JOURNAL OF

SYMPLECTIC GEOMETRY

Volume 3, Number 3, 313-340, 2005

\title{
PACKING SYMPLECTIC MANIFOLDS BY HAND
}

\author{
FELIX SCHLENK
}

We construct explicit maximal symplectic packings of minimal rational and ruled symplectic 4 -manifolds by few balls in a very simple way.

\section{Introduction}

Consider a connected $2 n$-dimensional symplectic manifold $(M, \omega)$ of finite volume $\operatorname{Vol}(M, \omega)=\frac{1}{n !} \int_{M} \omega^{n}$, and let $B^{2 n}(a)$ be the open ball of radius $\sqrt{a / \pi}$ in standard symplectic space $\left(\mathbb{R}^{2 n}, \omega_{0}\right)$. Then $\operatorname{Vol}\left(B^{2 n}(a), \omega_{0}\right)=$ $a^{n} / n$ !, as it should be. The $k$ th symplectic packing number $\left.\left.p_{k}(M, \omega) \in\right] 0,1\right]$ is defined as

$$
p_{k}(M, \omega)=\sup _{a} \frac{k \operatorname{Vol}\left(B^{2 n}(a), \omega_{0}\right)}{\operatorname{Vol}(M, \omega)}
$$

where the supremum is taken over all those $a$ for which the disjoint union $\coprod_{i=1}^{k} B^{2 n}(a)$ of $k$ equal balls symplectically embeds into $(M, \omega)$. We refer to $[\mathbf{1 8}]$ and to $[\mathbf{2 1}]$ for various motivations to determine or estimate these numbers. If $p_{k}(M, \omega)<1$, one says that there is a packing obstruction, and if $p_{k}(M, \omega)=1$, one says that $(M, \omega)$ admits a full packing by $k$ balls. The first examples of packing obstructions were found by Gromov [10], and many further packing obstructions and also some exact values of $p_{k}$ were obtained by McDuff and Polterovich in $[\mathbf{1 8}]$. Finally, Biran showed in $[\mathbf{5}, \mathbf{6}]$ that

$$
P(M, \omega):=\inf \left\{k_{0} \in \mathbb{N} \mid p_{k}(M, \omega)=1 \text { for all } k \geq k_{0}\right\}<\infty
$$

for an interesting class of closed symplectic 4-manifolds containing sphere bundles over a surface and for all closed symplectic 4-manifolds with $[\omega] \in$ $H^{2}(M ; \mathbb{Q})$.

Besides sporadic results on the first packing number $p_{1}$ and on packing numbers for ellipsoids in $[\mathbf{1 4}, \mathbf{2 3}]$, all known computations of packing numbers are contained in $[\mathbf{5}, \mathbf{6}, \mathbf{1 8}]$. We refer to Biran's excellent survey $[\mathbf{8}]$

for the methods used, and only mention that in $[\mathbf{5}, \mathbf{6}, \mathbf{1 8}]$ the problem of 
symplectically embedding $k$ equal balls into $(M, \omega)$ is first reformulated as the problem of deforming a symplectic form on the $k$-fold blow-up of $(M, \omega)$ along a certain family of cohomology classes, and that this problem is then solved using tools from classical algebraic geometry, Seiberg-Witten-Taubes theory, and Donaldson's symplectic submanifold theorem, respectively. As a consequence, the symplectic packings found are not explicit. For some of the symplectic manifolds considered in $[\mathbf{5}, \mathbf{6}, \mathbf{1 8}]$ and some values of $k$, explicit maximal symplectic packings were constructed by Karshon [11], Traynor [23], Kruglikov [12], and Maley, Mastrangeli, and Traynor [14]. In this article, we describe a very simple and explicit construction realizing the packing numbers $p_{k}(M, \omega)$ for those symplectic 4-manifolds $(M, \omega)$ and numbers $k$ considered in $[\mathbf{1 1}, \mathbf{1 2}, \mathbf{1 4}, \mathbf{2 3}]$, as well as for some other closed symplectic 4-manifolds and small values of $k$. To be more precise, we construct maximal packings different from those in $[\mathbf{1 1}, \mathbf{1 2}, \mathbf{1 4}, \mathbf{2 3}]$ of the 4-ball and of $\mathbb{C P}^{2}$ by $k \leq 6$ balls and by $l^{2}$ balls for each $l \in \mathbb{N}$, and of the product of two surfaces of equal area by $2 l^{2}$ balls. In addition, we construct maximal packings of $S^{2} \times S^{2}$ by $k \leq 6$ balls for all symplectic structures and by 7 balls for some symplectic structures, as well as maximal packings of the non-trivial bundle $S^{2} \ltimes S^{2}$ by $k \leq 5$ balls for all symplectic structures and by 6 balls for some symplectic structures. In the range of $k$ for which these constructions fail to give maximal packings, they give a feeling that the balls in the packings from $[\mathbf{5}, \mathbf{6}, \mathbf{1 8}]$ must be "wild". Our symplectic packings are simply obtained via products $\alpha_{1} \times \alpha_{2}$ of suitable area preserving diffeomorphisms between a disc and a rectangle. Taking $n$-fold products, we shall also construct explicit maximal packings of the $2 n$-ball and $\mathbb{C P}^{n}$ and of $\mathbb{C P}^{n} \backslash \mathbb{R} \mathbb{P}^{n}$ by $k \leq 2^{n}$ equal balls and explicit full packings of these spaces by $l^{n}$, respectively, $2^{l n}$ equal balls for each $l \in \mathbb{N}$ in a most simple way.

We finally consider a relative packing problem. A middle-dimensional submanifold $L$ of a symplectic manifold $(M, \omega)$ is called Lagrangian if $\omega$ vanishes on $T L$. Examples are $B^{2 n}(\pi) \cap \mathbb{R}^{n}(x)$ in $B^{2 n}(\pi)$ and $\mathbb{R} \mathbb{P}^{n}$ in $\mathbb{C P}^{n}$. Given a connected $2 n$-dimensional symplectic manifold $(M, \omega)$ of finite volume and a Lagrangian submanifold $L$ of $M$, we define the $k$ th relative symplectic packing number $\left.\left.p_{k}(M, L, \omega) \in\right] 0,1\right]$ as

$$
p_{k}(M, L, \omega)=\sup _{a} \frac{k \operatorname{Vol}\left(B^{2 n}(a), \omega_{0}\right)}{\operatorname{Vol}(M, \omega)}
$$

where now the supremum is taken over all those $a$ for which there exists a symplectic embedding $\coprod_{i=1}^{k} \varphi_{i}: \coprod_{i=1}^{k} B^{2 n}(a) \hookrightarrow(M, \omega)$ such that $\varphi_{i}^{-1}(L)=$ $B^{2 n}(a) \cap \mathbb{R}^{n}(x)$. In other words, we allow only for symplectic embeddings which map $B^{2 n}(a) \cap \mathbb{R}^{n}(x)$ to $L$ and $B^{2 n}(a) \backslash \mathbb{R}^{n}(x)$ to $M \backslash L$. For $k=1$, such relative symplectic embeddings were introduced recently by Barraud and Cornea in $[\mathbf{1}, \mathbf{2}]$, see in particular $[\mathbf{2}$, p. 32]. We refer to their work 
for motivations to study $p_{1}(M, L, \omega)$. It is not yet clear whether the methods from $[\mathbf{5}, \mathbf{6}, \mathbf{1 8}]$ can be adopted to study the relative packing numbers $p_{k}(M, L, \omega)$. Notice that one always has

$$
p_{k}(M, L, \omega) \leq p_{k}(M, \omega) .
$$

We shall only look at the Lagrangian submanifolds $B^{2 n}(\pi) \cap \mathbb{R}^{n}(x)$ in $B^{2 n}(\pi)$ and $\mathbb{R P}^{n}$ in $\mathbb{C P}^{n}$, and shall notice that our explicit maximal packings of $B^{2 n}(\pi)$ and $\mathbb{C P}^{n}$ are in fact relative packings for these Lagrangian submanifolds, so that for these examples equality holds in (2).

In Section 2, we collect the packing numbers of interest to us. In Section 3, we construct our maximal packings of symplectic 4-manifolds, and in Section 4, we construct our packings of the $2 n$-ball and of $\mathbb{C P} \mathbb{P}^{n} \backslash \mathbb{R} \mathbb{P}^{n}$. In Section 5, we verify that our maximal packings of $B^{2 n}(\pi)$ and $\mathbb{C P}^{n}$ are relative packings for $B^{2 n}(\pi) \cap \mathbb{R}^{n}(x)$ and $\mathbb{R} \mathbb{P}^{n}$.

Balls will always be endowed with the standard symplectic form $\omega_{0}=$ $\sum_{i=1}^{n} d x_{i} \wedge d y_{i}$. Since the packing numbers $p_{k}\left(B^{2 n}(a), \omega_{0}\right)$ do not depend on $a$, we shall usually pack the unit ball $B^{2 n}:=\left(B^{2 n}(\pi), \omega_{0}\right)$.

\section{Packing numbers}

In this section, we list the known packing numbers of interest to us and compute $p_{k}$ for the nontrivial sphere bundles over Riemann surfaces for $k \leq 7$.

2.1. The packing numbers of the 4 -ball and $\mathbb{C P}^{2}$. Let $\omega_{S F}$ be the unique U(3)-invariant Kähler form on $\mathbb{C P}^{2}$ whose integral over $\mathbb{C P}^{1}$ equals 1 . According to a result of Taubes $[\mathbf{2 2}]$, every symplectic form on $\mathbb{C P}^{2}$ is diffeomorphic to $a \omega_{S F}$ for some $a>0$. In view of the symplectomorphism

$$
\left(B^{4}(\pi), \omega_{0}\right) \rightarrow\left(\mathbb{C P}^{2} \backslash \mathbb{C P}^{1}, \pi \omega_{S F}\right), \quad z=\left(z_{1}, z_{2}\right) \mapsto\left[z_{1}: z_{2}: \sqrt{1-|z|^{2}}\right]
$$

further discussed in $\left[\mathbf{1 9}\right.$, Example 7.14] we have $p_{k}\left(B^{4}\right) \leq p_{k}\left(\mathbb{C P}^{2}\right)$ for all $k$. It is shown in [18, Remark 2.1.E] that in fact

$$
p_{k}\left(B^{4}\right)=p_{k}\left(\mathbb{C P}^{2}\right) \text { for all } k \text {. }
$$

A complete list of these packing numbers was obtained in [5] (see Table 1).

\begin{tabular}{cccccccccc}
\hline$k$ & 1 & 2 & 3 & 4 & 5 & 6 & 7 & 8 & $\geq 9$ \\
\hline$p_{k}$ & 1 & $\frac{1}{2}$ & $\frac{3}{4}$ & 1 & $\frac{20}{25}$ & $\frac{24}{25}$ & $\frac{63}{64}$ & $\frac{288}{289}$ & 1 \\
\hline \multicolumn{1}{c}{ Table 1. } & $p_{k}\left(B^{4}\right)=p_{k}\left(\mathbb{C P}^{2}\right)$
\end{tabular}


Explicit maximal packings were found by Karshon [11] for $k \leq 3$ and by Traynor [23] for $k \leq 6$ and $k=l^{2}(l \in \mathbb{N})$. We will give even simpler maximal packings for these values of $k$ in Section 3.2.

2.2. The packing numbers of ruled symplectic 4-manifolds. Denote by $\Sigma_{g}$ the closed orientable surface of genus $g$. There are exactly two orientable $S^{2}$-bundles with base $\Sigma_{g}$, namely the trivial bundle $\pi: \Sigma_{g} \times S^{2} \rightarrow \Sigma_{g}$ and the nontrivial bundle $\pi: \Sigma_{g} \ltimes S^{2} \rightarrow \Sigma_{g}$, see [19, Lemma 6.9]. Such a manifold $M$ is called a ruled surface. A symplectic form $\omega$ on a ruled surface is called compatible with the given ruling $\pi$ if it restricts on each fibre to a symplectic form. Such a symplectic manifold is then called a ruled symplectic 4-manifold. It is known that every symplectic structure on a ruled surface is diffeomorphic to a form compatible with the given ruling $\pi$ via a diffeomorphism which acts trivially on homology, and that two cohomologous symplectic forms compatible with the same ruling are isotopic [13]. A symplectic form $\omega$ on a ruled surface $M$ is thus determined up to diffeomorphism by the class $[\omega] \in H^{2}(M ; \mathbb{R})$. In order to describe the set of cohomology classes realized by (compatible) forms on $M$, we fix an orientation of $\Sigma_{g}$ and an orientation of the fibres of the given ruled surface $M$. These orientations determine an orientation of $M$ in a natural way, see below. We say that a compatible symplectic form $\omega$ is admissible if its restriction to each fibre induces the given orientation and if $\omega$ induces the natural orientation on $M$. Notice that every symplectic form on $M$ is diffeomorphic to an admissible form for a suitable choice of orientations of $\Sigma_{g}$ and the fibres.

Consider first the trivial bundle $\Sigma_{g} \times S^{2}$. The two classes $B=\left[\Sigma_{g} \times p t\right]$ and $F=\left[p t \times S^{2}\right]$ form a basis of $H^{2}(M ; \mathbb{Z})$. Here, and henceforth, we identify homology and cohomology via Poincaré duality. The natural orientation of $\Sigma_{g} \times S^{2}$ is such that $B \cdot F=1$. A cohomology class $C=b B+a F$ can be represented by an admissible form if and only if $C \cdot F>0$ and $C \cdot C>0$, i.e.,

$$
a>0 \text { and } b>0,
$$

standard representatives being split forms. We write $\Sigma_{g}(a) \times S^{2}(b)$ for this ruled symplectic 4-manifold.

In case of the nontrivial bundle $\Sigma_{g} \ltimes S^{2}$, a basis of $H^{2}\left(\Sigma_{g} \ltimes S^{2} ; \mathbb{Z}\right)$ is given by $\{A, F\}$, where $A$ is the class of a section with self-intersection number -1 and $F$ is the fibre class. The homology classes of sections of $\Sigma_{g} \ltimes S^{2}$ of self-intersection number $k$ are $A_{k}=A+\frac{k+1}{2} F$ with $k$ odd. The natural orientation of $\Sigma_{g} \ltimes S^{2}$ is such that $A_{k} \cdot F=A \cdot F=1$ for all $k$. Set $B=A+F / 2$. Then $\{B, F\}$ is a basis of $H^{2}\left(\Sigma_{g} \ltimes S^{2} ; \mathbb{R}\right)$ with $B \cdot B=F \cdot F=0$ and $B \cdot F=1$. As for the trivial bundle, the necessary condition for a cohomology class $b B+a F$ to be representable by an admissible form is $a>0$ and $b>0$. It turns out that this condition is sufficient only if $g \geq 1$. 
A cohomology class $b B+a F$ can be represented by an admissible form if and only if

$$
\begin{array}{ll}
a>b / 2>0 & \text { if } g=0, \\
a>0 \text { and } b>0 & \text { if } g \geq 1,
\end{array}
$$

see [19, Theorem 6.11]. We write $\left(\Sigma_{g} \ltimes S^{2}, \omega_{a b}\right)$ for this ruled symplectic 4-manifold. A "standard Kähler form" in the class $\left[\omega_{a b}\right]$ is explicitly constructed in [16, Section 3] and [19, Exercise 6.14]. When constructing our explicit symplectic packings, it will always be clear which symplectic form in $\left[\omega_{a b}\right]$ is chosen.

We begin with the trivial sphere bundle over the sphere.

Proposition 2.1. Assume that $a \geq b$. Abbreviate $p_{k}=p_{k}\left(S^{2}(a) \times S^{2}(b)\right)$, and denote by $\lceil x\rceil$ the minimal integer which is greater than or equal to $x$. Then

$$
p_{k}=\frac{k}{2} \frac{b}{a} \quad \text { if }\left\lceil\frac{k}{2}\right\rceil \frac{b}{a} \leq 1 .
$$

Moreover,

$$
\begin{gathered}
\left.\left.p_{1}=\frac{b}{2 a}, \quad p_{2}=\frac{b}{a}, \quad p_{3}=\frac{3}{2 a b}\left\{b, \frac{a+b}{3}\right\}^{2} \text { on }\right] 0, \frac{1}{2}, 1\right], \\
\left.\left.p_{4}=\frac{4}{3} p_{3}, \quad p_{5}=\frac{5}{2 a b}\left\{b, \frac{a+2 b}{5}\right\}^{2} \text { on }\right] 0, \frac{1}{3}, 1\right], \\
\left.\left.p_{6}=\frac{3}{a b}\left\{b, \frac{a+2 b}{5}, \frac{2 a+2 b}{7}\right\}^{2} \text { on }\right] 0, \frac{1}{3}, \frac{3}{4}, 1\right], \\
\left.\left.p_{7}=\frac{7}{2 a b}\left\{b, \frac{a+3 b}{7}, \frac{3 a+4 b}{13}, \frac{4 a+4 b}{15}\right\}^{2} \text { on }\right] 0, \frac{1}{4}, \frac{8}{11}, \frac{7}{8}, 1\right] .
\end{gathered}
$$

In particular, for $k \leq 7$ we have $p_{k}\left(S^{2}(a) \times S^{2}(b)\right)=1$ exactly for the values $\left(k=2, \frac{b}{a}=1\right),\left(k=4, \frac{b}{a}=\frac{1}{2}\right),\left(k=6, \frac{b}{a}=\frac{1}{3}\right),\left(k=6, \frac{b}{a}=\frac{3}{4}\right)$ and $\left(k=7, \frac{b}{a}=\frac{7}{8}\right)$.

We explain our notation by an example: $p_{3}=\frac{3}{2 a b} b^{2}$ if $0<\frac{b}{a} \leq \frac{1}{2}$ and $p_{3}=\frac{3}{2 a b}\left(\frac{a+b}{3}\right)^{2}$ if $\frac{1}{2} \leq \frac{b}{a} \leq 1$.

In Section 3.3.1, we will construct explicit maximal packings of the symplectic manifold $S^{2}(a) \times S^{2}(b)$ for all $k$ with $\left\lceil\frac{k}{2}\right\rceil \frac{b}{a} \leq 1$, for $k \leq 6$ and $0<b \leq a$ arbitrary, and for $k=7$ and $0<\frac{b}{a} \leq \frac{3}{5}$, as well as explicit full packings for $k=2 m l^{2}$ if $a=m b(l, m \in \mathbb{N})$. These explicit packings will give to the above quantities a transparent geometric meaning.

The following corollary slightly refines Corollary 5.B of [5].

Corollary 2.2. We have $\max \left(2 \frac{a}{b}, 8\right) \leq P\left(S^{2}(a) \times S^{2}(b)\right) \leq 8 \frac{a}{b}$ except possibly for $\frac{b}{a}=\frac{7}{8}$, in which case $P\left(S^{2}(a) \times S^{2}(b)\right) \in\{7,8,9\}$. For $S^{2}(1) \times S^{2}(1)$ we thus have the following table: 


\begin{tabular}{ccccccccc}
\hline$k$ & 1 & 2 & 3 & 4 & 5 & 6 & 7 & $\geq 8$ \\
\hline$p_{k}$ & $\frac{1}{2}$ & 1 & $\frac{2}{3}$ & $\frac{8}{9}$ & $\frac{9}{10}$ & $\frac{48}{49}$ & $\frac{224}{225}$ & 1 \\
\hline
\end{tabular}

Table 2. $p_{k}\left(S^{2}(1) \times S^{2}(1)\right)$

Proposition 2.3. Assume that $a>\frac{b}{2}>0$. Abbreviate $p_{k}=p_{k}\left(S^{2} \ltimes S^{2}, \omega_{a b}\right)$, and set $\langle k\rangle=k$ if $k$ is odd and $\langle k\rangle=k+1$ if $k$ is even. Then

$$
p_{k}=\frac{k}{2} \frac{b}{a} \quad \text { if } \frac{\langle k\rangle}{2} \frac{b}{a} \leq 1
$$

Moreover,

$$
\begin{gathered}
\left.p_{1}=\frac{b}{2 a}, \quad p_{2}=\frac{1}{a b}\left\{b, \frac{2 a+b}{4}\right\}^{2} \text { on }\right] 0, \frac{2}{3}, 2[ \\
\left.p_{3}=\frac{3}{2} p_{2}, \quad p_{4}=\frac{2}{a b}\left\{b, \frac{2 a+3 b}{8}\right\}^{2} \text { on }\right] 0, \frac{2}{5}, 2[, \\
\left.p_{5}=\frac{5}{2 a b}\left\{b, \frac{2 a+3 b}{8}, \frac{2 a+b}{5}\right\}^{2} \text { on }\right] 0, \frac{2}{5}, \frac{6}{7}, 2[, \\
\left.p_{6}=\frac{3}{a b}\left\{b, \frac{2 a+5 b}{12}, \frac{2 a+2 b}{7}, \frac{2 a+b}{5}\right\}^{2} \text { on }\right] 0, \frac{2}{7}, \frac{10}{11}, \frac{4}{3}, 2[, \\
p_{7}=\frac{7}{2 a b}\left\{b, \frac{2 a+5 b}{12}, \frac{6 a+9 b}{28}, \frac{4 a+4 b}{15}, \frac{4 a+3 b}{13}, \frac{6 a+3 b}{16}\right\}^{2} \\
\text { on }] 0, \frac{2}{7}, \frac{1}{2}, \frac{22}{23}, \frac{8}{7}, \frac{14}{9}, 2[.
\end{gathered}
$$

In particular, for $k \leq 7$, we have $p_{k}\left(S^{2} \ltimes S^{2}, \omega_{a b}\right)=1$ exactly for the values $\left(k=3, \frac{b}{a}=\frac{2}{3}\right),\left(k=5, \frac{b}{a}=\frac{2}{5}\right),\left(k=6, \frac{b}{a}=\frac{4}{3}\right),\left(k=7, \frac{b}{a}=\frac{2}{7}\right)$, $\left(k=7, \frac{b}{a}=\frac{8}{7}\right)$ and $\left(k=7, \frac{b}{a}=\frac{14}{9}\right)$.

In Section 3.3.2, we will construct explicit maximal packings of the symplectic manifold $\left(S^{2} \ltimes S^{2}, \omega_{a b}\right)$ for all $k$ with $\frac{\langle k\rangle}{2} \frac{b}{a} \leq 1$, for $k \leq 5$ and $0<\frac{b}{2}<a$ arbitrary, and for $k=6$ and $\left.\left.\frac{b}{a} \in\right] 0, \frac{2}{3}\right] \cup\left[\frac{4}{3}, 2[\right.$. Moreover, given $\omega_{a b}$ with $\frac{b}{a}=\frac{2 l}{2 m-l}$ for some $l, m \in \mathbb{N}$ with $m>l$, we will construct explicit full packings of $\left(S^{2} \ltimes S^{2}, \omega_{a b}\right)$ by $l(2 m-l)$ balls.

Corollary 2.4. $\max \left(2 \frac{a}{b}, 8\right) \leq P\left(S^{2} \ltimes S^{2}, \omega_{a b}\right) \leq\left\{\begin{array}{cl}\frac{8 a}{b} & \text { if } b \leq a \\ \frac{8 a b}{(2 a-b)^{2}} & \text { if } b \geq a\end{array}\right.$ except possibly for $\frac{b}{a} \in\left\{\frac{2}{7}, \frac{8}{7}, \frac{14}{9}\right\}$, in which case the lower bound for the number $P\left(S^{2} \ltimes S^{2}, \omega_{a b}\right)$ is 7 . For $\left(S^{2} \ltimes S^{2}, \omega_{11}\right)$ we thus have the following table: 


\begin{tabular}{ccccccccc}
\hline$k$ & 1 & 2 & 3 & 4 & 5 & 6 & 7 & $\geq 8$ \\
\hline$p_{k}$ & $\frac{1}{2}$ & $\frac{9}{16}$ & $\frac{27}{32}$ & $\frac{25}{32}$ & $\frac{9}{10}$ & $\frac{48}{49}$ & $\frac{14}{15}$ & 1 \\
\hline
\end{tabular}

Table 3. $p_{k}\left(S^{2} \ltimes S^{2}, \omega_{11}\right)$

Proposition 2.5. Let $g \geq 1$ and let $a>0$ and $b>0$. Then

$$
p_{k}\left(\Sigma_{g}(a) \times S^{2}(b)\right)=p_{k}\left(\Sigma_{g} \ltimes S^{2}, \omega_{a b}\right)=\min \left\{1, \frac{k}{2} \frac{b}{a}\right\} .
$$

In particular, $P\left(\Sigma_{g}(a) \times S^{2}(b)\right)=P\left(\Sigma_{g} \ltimes S^{2}, \omega_{a b}\right)=\left\lceil\frac{2 a}{b}\right\rceil$.

In Section 3.3.3, we will construct explicit maximal packings of the symplectic manifolds $\Sigma_{g}(a) \times S^{2}(b)$ and $\left(\Sigma_{g} \ltimes S^{2}, \omega_{a b}\right)$ for all $k$ with $\left\lceil\frac{k}{2}\right\rceil \frac{b}{a} \leq 1$ and explicit full packings for $k=2 m l^{2}$, if $a=m b$ or $b=m a(l, m \in \mathbb{N})$.

2.3. The packing numbers of the $2 n$-ball and of $\mathbb{C P}^{n} \backslash \mathbb{R} \mathbb{P}^{n}$. As in dimension 4 , we denote by $\omega_{S F}$ the unique $\mathrm{U}(n+1)$-invariant Kähler form on $\mathbb{C P}^{n}$ whose integral over $\mathbb{C P}^{1}$ equals 1 . The symplectomorphism (3) generalizes to the symplectomorphism $\left(B^{2 n}(\pi), \omega_{0}\right) \rightarrow\left(\mathbb{C P}^{n} \backslash \mathbb{C P}^{n-1}, \pi \omega_{S F}\right)$ defined by

$$
z=\left(z_{1}, \ldots, z_{n}\right) \mapsto\left[z_{1}: \cdots: z_{n}: \sqrt{1-|z|^{2}}\right],
$$

and $p_{k}\left(B^{2 n}\right)=p_{k}\left(\mathbb{C P}^{n}, \omega_{S F}\right)$ for all $k$, see $[\mathbf{1 8}$, Remark 2.1.E]. While the packing numbers $p_{k}\left(B^{4}\right)=p_{k}\left(\mathbb{C P}^{2}\right)$ are listed in Table 1 above, for $n \geq 3$ the only known results are

$$
\begin{array}{rll}
p_{k}\left(B^{2 n}\right)=p_{k}\left(\mathbb{C P}^{n}\right) & =\frac{k}{2^{n}} & \text { for } 2 \leq k \leq 2^{n}, \\
p_{l^{n}}\left(B^{2 n}\right)=p_{l^{n}}\left(\mathbb{C} P^{n}\right) & =1 & \text { for all } l \in \mathbb{N},
\end{array}
$$

see $[\mathbf{1 0}]$ and $[\mathbf{1 8}$, Corollary 1.5.C and 1.6.B]. We shall realize these packing numbers in Section 4.1 by very simple explicit packings.

Let $\mathbb{R} \mathbb{P}^{n} \subset \mathbb{C P}{ }^{n}$ be the $n$-dimensional real projective space embedded in the usual way (as the fixed point set of the canonical conjugation of $\left.\mathbb{C P}^{n}\right)$. Biran proved in $[7]$ that $p_{1}\left(\mathbb{C P}^{n} \backslash \mathbb{R P}^{n}\right)=1 / 2^{n}$. Therefore, $p_{k}\left(\mathbb{C P}^{n} \backslash \mathbb{R P}^{n}\right) \leq k / 2^{n}$ for all $k \leq 2^{n}$. In view of the symplectomorphism (5), the "pierced ball" $B^{2 n}(\pi) \backslash \mathbb{R}^{n}(x)$ in $\left(\mathbb{R}^{2 n}, \omega_{0}\right)$ symplectically embeds into $\left(\mathbb{C P}^{n} \backslash \mathbb{R} \mathbb{P}^{n}, \pi \omega_{S F}\right)$. In Section 4.2 , we shall construct explicit full packings of $B^{2 n}(\pi) \backslash \mathbb{R}^{n}(x)$ by $2^{l n}$ equal balls for all $l \in \mathbb{N}$. It follows 
that

(9) $p_{2^{l n}}\left(B^{2 n} \backslash \mathbb{R}^{n}(x)\right)=p_{2^{l n}}\left(\mathbb{C P}^{n} \backslash \mathbb{R P}^{n}\right)=1 \quad$ for all $l \in \mathbb{N}$.

Remark 2.6. For $n=2$, Biran's work provides more information. It is shown in $[7]$ that $\left(\mathbb{C P}^{2} \backslash \mathbb{R P}^{2}, \omega_{S F}\right)$ is symplectomorphic to a symplectic disc bundle over the smooth quadric $S^{2} \cong \Sigma=\left\{z_{1}^{2}+z_{2}^{2}+z_{3}^{2}=0\right\}$ such that $\Sigma$ has area 2 and the fibres have area $1 / 2$. According to [6], this disc bundle can be compactified in a symplectic way to an $S^{2}$-bundle over $\Sigma$ whose packing numbers agree (up to $\epsilon$ ). Since $\Sigma \cdot \Sigma=4$, this is the trivial $S^{2}$-bundle over $S^{2}$, and the symplectic form in question is the one of $S^{2}(1) \times S^{2}\left(\frac{1}{2}\right)$. Proposition 2.1 and Corollary 2.2 thus yield Table 4 and $8 \leq P\left(\mathbb{C P}^{2} \backslash \mathbb{R P}^{2}\right) \leq 16$.

\begin{tabular}{cccccccc}
\hline$k$ & 1 & 2 & 3 & 4 & 5 & 6 & 7 \\
\hline$p_{k}$ & $\frac{1}{4}$ & $\frac{1}{2}$ & $\frac{3}{4}$ & 1 & $\frac{20}{25}$ & $\frac{24}{25}$ & $\frac{25}{28}$ \\
\hline
\end{tabular}

Table 4. $p_{k}\left(\mathbb{C P}^{2} \backslash \mathbb{R P}^{2}\right)$ for $k \leq 7$

Comparing with Table 1 , we see that $p_{k}\left(\mathbb{C P}^{2} \backslash \mathbb{R} \mathbb{P}^{2}\right)=p_{k}\left(\mathbb{C P}^{2}\right)$ except for $k \in\{1,7\}$ and possibly for $k \in\{8, \ldots, 15\}$. Notice that

$$
B^{4}(\pi) \backslash \mathbb{R}^{2}(x)=\left(\mathbb{C P}^{2} \backslash \mathbb{R P}^{2}\right) \backslash\left(\mathbb{C P}^{1} \backslash \mathbb{R P}^{1}\right) .
$$

In view of Remark 2.1.E in [18], it is thus plausible that $p_{k}\left(B^{4} \backslash \mathbb{R}^{2}(x)\right)=$ $p_{k}\left(\mathbb{C P}^{2} \backslash \mathbb{R P}^{2}\right)$ for all $k$. The identities (8) and (9) verify this for $k \leq 4$ and $k=4^{l}$.

In the remainder of this section, we prove Propositions 2.1, 2.3, and 2.5 and Corollaries 2.2 and 2.4. We assume the reader to be familiar with [5]. Set $N=\mathbb{C P}^{2}$, let $L=\left[\mathbb{C P}^{1}\right]$ be the positive generator of $H^{2}(N ; \mathbb{Z})$, let $\widetilde{N}_{k}$ be the complex blow-up of $\mathbb{C P}^{2}$ at $k$ points and let $D_{1}, \ldots, D_{k}$ be the classes of the exceptional divisors.

Proof of Proposition 2.1. Fix $0<b \leq a$ and set $p_{k}=p_{k}\left(S^{2}(a) \times S^{2}(b)\right)$. Biran [5, Theorem 6.1.A] showed that for any $k \in \mathbb{N}$,

$$
p_{k}=\min \left\{1, \frac{k}{2 a b} \inf \left(\frac{a n_{1}+b n_{2}}{2 n_{1}+2 n_{2}-1}\right)^{2}\right\}
$$

where the infimum is taken over all $n_{1}, n_{2} \in \mathbb{N}_{0}:=\mathbb{N} \cup\{0\}$ for which the system of Diophantine equations

$$
\left.\begin{array}{rl}
2 n_{1} n_{2} & =\sum_{i=1}^{k} m_{i}^{2}-1 \\
2 n_{1}+2 n_{2} & =\sum_{i=1}^{k} m_{i}+1
\end{array}\right\}
$$


has a solution $\left(m_{1}, \ldots, m_{k}\right) \in \mathbb{N}_{0}^{k}$. It is easy to see that the only solutions of (11.1) are $\left(n_{1}=0, n_{2}=1, m_{1}=1\right)$ and $\left(n_{1}=1, n_{2}=0, m_{1}=1\right)$, which yields $p_{1}=\frac{b}{2 a}$. This implies that $p_{k} \leq \frac{k}{2} \frac{b}{a}$ for all $0<b \leq a$ and all $k$, and that the reverse inequality holds true whenever $\left\lceil\frac{k}{2}\right\rceil \frac{b}{a} \leq 1$ will be shown in Section 3.3.1. In order to compute $p_{k}$ for $2 \leq k \leq 7$, let $\widetilde{M}_{k}$ be the complex blow-up of $S^{2} \times S^{2}$ at $k$ points and let $E_{1}, \ldots, E_{k}$ be the classes of the exceptional divisors. Recall that we chose the basis of $H^{2}\left(S^{2} \times S^{2} ; \mathbb{Z}\right)$ to be $\left.\left\{B=\left[S^{2} \times p t\right], F=p t \times S^{2}\right]\right\}$. The solutions of (11.k) correspond to the exceptional elements $E=n_{1} B+n_{2} F-\sum_{i=1}^{k} m_{i} E_{i} \in H_{2}\left(\widetilde{M}_{k} ; \mathbb{Z}\right)$ with $n_{1}, n_{2}, m_{1}, \ldots, m_{k} \geq 0$.

Observe now that for $k \geq 1, \widetilde{M}_{k}$ is diffeomorphic to $\widetilde{N}_{k+1}$ via a diffeomorphism under which the classes $L, D_{1}, D_{2}, D_{3}, \ldots, D_{k+1}$ correspond to the classes $B+F-E_{1}, B-E_{1}, F-E_{1}, E_{2}, \ldots, E_{k}$, respectively, cf. Figure 7 below. The exceptional elements in $H_{2}\left(\widetilde{N}_{k+1} ; \mathbb{Z}\right)$ for $k \leq 7$ are listed in $[\mathbf{9}$, p. 35]. The values $p_{k}$ for $2 \leq k \leq 7$ are now obtained by evaluating this list in $(10)$.

Proof of Corollary 2.2. The estimates $2 \frac{a}{b} \leq P\left(S^{2}(a) \times S^{2}(b)\right) \leq 8 \frac{a}{b}$ are proved in [5, Corollary B.5]. The claim now follows from the last statement in Proposition 2.1.

Proof of Proposition 2.3. Fix $a>\frac{b}{2}>0$ and set $M=S^{2} \ltimes S^{2}$ and $p_{k}=$ $p_{k}\left(M, \omega_{a b}\right)$. With $\alpha=a-\frac{b}{2}$ and $\beta=b$ the condition $a>\frac{b}{2}>0$ becomes $\alpha>0$ and $\beta>0$. Recall that $\omega_{a b}=b B+a F=\beta A+(\alpha+\beta) F$, where $\{A, F\}$ is a basis of $H_{2}(M ; \mathbb{Z})$ with $A \cdot A=-1, A \cdot F=1$, and $F \cdot F=0$. Let $\Theta: \widetilde{M}_{k} \rightarrow M$ be the complex blow-up of $M$ at $k$ points and let $E_{1}, \ldots, E_{k}$ be the classes of the exceptional divisors. The first Chern class of $M$ is $c_{1}=2 A+3 F$, so that the first Chern class of $\widetilde{M}_{k}$ is $\tilde{c}_{1}=2 A+3 F-\sum_{i=1}^{k} E_{i}$. Let $E=n_{1} A+n_{2} F-\sum_{i=1}^{k} m_{i} E_{i}$ be an exceptional element in $H_{2}\left(\widetilde{M}_{k} ; \mathbb{Z}\right)$ with $m_{1}, \ldots, m_{k} \geq 0$, that is, $\left(n_{1}, n_{2}, m_{1}, \ldots, m_{k}\right) \in \mathbb{Z}^{2} \times \mathbb{N}_{0}^{k}$ is a solution of the system of Diophantine equations

$$
\left.\begin{array}{rl}
n_{1}\left(2 n_{2}-n_{1}\right) & =\sum_{i=1}^{k} m_{i}^{2}-1 \\
n_{1}+2 n_{2} & =\sum_{i=1}^{k} m_{i}+1
\end{array}\right\} .
$$

Suppose that $\widetilde{\omega}_{a b}$ is a symplectic form on $\widetilde{M}_{k}$ such that $\left[\widetilde{\omega}_{a b}\right]=\left[\Theta^{*} \omega_{a b}\right]-$ $\epsilon \sum_{i=1}^{k} E_{i}$. We claim that for $\epsilon>0$ small enough, $\widetilde{\omega}_{a b}\left(\tilde{c}_{1}+E\right)>0$. Indeed, since $m_{1}, \ldots, m_{k} \geq 0,(12 . \mathrm{k})$ implies that $n_{1}, n_{2} \geq 0$. Hence

$$
\widetilde{\omega}_{a b}\left(\tilde{c}_{1}+E\right)=\alpha\left(2+n_{1}\right)+\beta\left(3+n_{2}\right)-\epsilon \sum_{i=1}^{k}\left(1+m_{i}\right)
$$

is positive for $\epsilon$ small enough. 
It now follows exactly as in the proof of Theorem 6.1.A in [5] that for any $k \in \mathbb{N}$,

$$
p_{k}=\min \left\{1, \frac{k}{\beta(2 \alpha+\beta)} \inf \left(\frac{\alpha n_{1}+\beta n_{2}}{n_{1}+2 n_{2}-1}\right)^{2}\right\}
$$

where the infimum is taken over all $n_{1}, n_{2} \in \mathbb{N}_{0}$ for which (12.k) has a solution $\left(m_{1}, \ldots, m_{k}\right) \in \mathbb{N}_{0}^{k}$.

Observe now that for $k \geq 0, \widetilde{M}_{k}$ is diffeomorphic to $\widetilde{N}_{k+1}$ via a diffeomorphism under which the classes $L, D_{1}, D_{2}, \ldots, D_{k+1}$ correspond to the classes $A+F, A, E_{1}, \ldots, E_{k}$, respectively, see [19, Example 7.4]. Evaluating the list in $[\mathbf{9}]$ in (11) we obtain

$$
\begin{gathered}
\left.p_{1}=\frac{\beta}{2 \alpha+\beta}, \quad p_{2}=\frac{2}{\beta(2 \alpha+\beta)}\left\{\beta, \frac{\alpha+\beta}{2}\right\}^{2} \text { on }\right] 0,1, \infty[ \\
\left.p_{3}=\frac{3}{2} p_{2}, \quad p_{4}=\frac{4}{\beta(2 \alpha+\beta)}\left\{\beta, \frac{\alpha+2 \beta}{4}\right\}^{2} \text { on }\right] 0, \frac{1}{2}, \infty[ \\
\left.p_{5}=\frac{5}{\beta(2 \alpha+\beta)}\left\{\beta, \frac{\alpha+2 \beta}{4}, \frac{2 \alpha+2 \beta}{5}\right\}^{2} \text { on }\right] 0, \frac{1}{2}, \frac{3}{2}, \infty[ \\
\left.p_{6}=\frac{6}{\beta(2 \alpha+\beta)}\left\{\beta, \frac{\alpha+3 \beta}{6}, \frac{2 \alpha+3 \beta}{7}, \frac{2 \alpha+2 \beta}{5}\right\}^{2} \text { on }\right] 0, \frac{1}{3}, \frac{5}{3}, 4, \infty[ \\
p_{7}=\frac{7}{\beta(2 \alpha+\beta)}\left\{\beta, \frac{\alpha+3 \beta}{6}, \frac{3 \alpha+6 \beta}{14}, \frac{4 \alpha+6 \beta}{15}, \frac{4 \alpha+5 \beta}{13}, \frac{3 \alpha+3 \beta}{8}\right\}^{2} \\
\text { on }] 0, \frac{1}{3}, \frac{2}{3}, \frac{11}{6}, \frac{8}{3}, 7, \infty[.
\end{gathered}
$$

Replacing $\alpha$ by $a-\frac{b}{2}$ and $\beta$ by $b$, we finally obtain the values $p_{k}$ for $1 \leq k \leq 7$ as stated in Proposition 2.3. The identity $p_{1}=\frac{b}{2 a}$ implies that $p_{k} \leq \frac{k}{2} \frac{b}{a}$ for all $0<\frac{b}{2}<a$ and all $k$. That the reverse inequality holds true whenever $\frac{\langle k\rangle}{2} \frac{b}{a} \leq 1$ will be shown in Section 3.3.2.

Proof of Corollary 2.4. Since $p_{1}=\frac{b}{2 a}$, we have that $P\left(S^{2} \ltimes S^{2}, \omega_{a b}\right) \geq \frac{2 a}{b}$, and the last statement in Proposition 2.3 shows that $P\left(S^{2} \ltimes S^{2}, \omega_{a b}\right) \geq 8$ if $\frac{b}{a} \notin\left\{\frac{2}{7}, \frac{8}{7}, \frac{14}{9}\right\}$ and $P\left(S^{2} \ltimes S^{2}, \omega_{a b}\right) \geq 7$ if $\frac{b}{a} \in\left\{\frac{2}{7}, \frac{8}{7}, \frac{14}{9}\right\}$. Next, set

$$
d_{\alpha \beta}=\inf \frac{\alpha n_{1}+\beta n_{2}}{n_{1}+2 n_{2}-1}
$$

where the infimum is taken over all nonnegative solutions $n_{1}, n_{2}, m_{1}, \ldots, m_{k}$ of (12.k). We claim that $d_{\alpha \beta} \geq \min \left\{\alpha, \frac{\beta}{2}\right\}$. Indeed, (12.k) has no solution for $n_{1}=n_{2}=0$. Moreover, if $m_{1}=\cdots=m_{k}=0$, then $n_{1}=1$ and $n_{2}=0$, and the corresponding quotient is infinite. We may thus assume that $2 n_{2} \geq n_{1}$. 
It is easy to see that for all $\left(n_{1}, n_{2}\right) \in \mathbb{N}_{0}^{2} \backslash\{(0,0)\}$ with $2 n_{2} \geq n_{1}$,

$$
\frac{\alpha n_{1}+\beta n_{2}}{n_{1}+2 n_{2}-1}>\min \left\{\alpha, \frac{\beta}{2}\right\} \text {. }
$$

Therefore,

$$
p_{k} \geq \min \left\{1, \frac{k}{\beta(2 \alpha+\beta)} \min \left(\alpha, \frac{\beta}{2}\right)^{2}\right\}
$$

and so

$$
P\left(M, \omega_{a b}\right) \leq \begin{cases}\frac{4(2 \alpha+\beta)}{\beta} & \text { if } \beta \leq 2 \alpha \\ \frac{\beta(2 \alpha+\beta)}{\alpha^{2}} & \text { if } \beta \geq 2 \alpha\end{cases}
$$

Replacing $\alpha$ by $a-\frac{b}{2}$ and $\beta$ by $b$ the claim follows.

Proof of Proposition 2.5. The statement for $\Sigma_{g}(a) \times S^{2}(b)$ was proved in [5, Theorem 6.1.A]. So, let $\left(M, \omega_{a b}\right)=\left(\Sigma_{g} \ltimes S^{2}, \omega_{a b}\right)$. We think of $M$ as the projectivization $\mathbb{P}\left(L_{1} \oplus \mathbb{C}\right) \stackrel{\pi}{\rightarrow} \Sigma_{g}$ of the complex rank two bundle $L_{1} \oplus \mathbb{C}$ over $\Sigma_{g}$, where $L_{1}$ is a holomorphic line bundle of Chern index 1 , and we endow $\mathbb{P}\left(L_{1} \oplus \mathbb{C}\right)$ with its canonical complex structure $J_{\text {can }}$. Let $\left(\widetilde{M}_{k}, \widetilde{J}_{\text {can }}\right)$ be the complex blow-up of $\left(\mathbb{P}\left(L_{1} \oplus \mathbb{C}\right), J_{\text {can }}\right)$ at $k$ generic points and let $\widetilde{\omega}_{a b}$ be a blow-up of $\omega_{a b}$. Finally, denote by $\mathcal{E}_{k}$ the set of homology classes of $\widetilde{M}_{k}$ which can be represented by $\widetilde{\omega}_{a b}$-symplectic exceptional spheres. We claim that

$$
\mathcal{E}_{k}\left(\widetilde{M}_{k}, \widetilde{\omega}_{a b}\right)=\left\{E_{1}, \ldots, E_{k}, F-E_{1}, \ldots, F-E_{k}\right\},
$$

where $E_{1}, \ldots, E_{k}$ are the classes of the exceptional divisors and where $F \in$ $H^{2}(M ; \mathbb{Z}) \subset H^{2}\left(\widetilde{M}_{k} ; \mathbb{Z}\right)$ is the fibre class. The analogous statement for $\Sigma_{g}(a) \times S^{2}(b)$ was proved by Biran [5], in the proof of his Corollary 5.C. His argument immediately applies to the twisted bundle and is repeated here for the sake of its beauty.

So, let $E=n_{1} A+n_{2} F-\sum_{i=1}^{k} m_{i} E_{i} \in \mathcal{E}_{k}$. Let $\mathcal{J}\left(\widetilde{\omega}_{a b}\right)$ be the space of $\widetilde{\omega}_{a b}$-tamed almost complex structures on $\widetilde{M}_{k}$ and let $\mathcal{J}_{E} \subset \mathcal{J}\left(\widetilde{\omega}_{a b}\right)$ be the subset of those $J$ for which there exist $J$-holomorphic $E$-spheres. It is known [4, Chapter V, proof of Lemma 2.C.2] that $\mathcal{J}_{E}$ contains a path-connected set which is open and dense in $\mathcal{J}\left(\widetilde{\omega}_{a b}\right)$. Let $\left\{\widetilde{J}_{t}\right\}_{0 \leq t \leq 1}$ be a smooth path in $\mathcal{J}\left(\widetilde{\omega}_{a b}\right)$ with $\left\{\widetilde{J}_{t}\right\}_{0 \leq t<1} \subset \mathcal{J}_{E}$ and $\widetilde{J}_{1}=\widetilde{J}_{\text {can }}$. Gromov's compactness theorem now shows that there exists a connected (but possibly cusp) $\widetilde{J}_{\text {can }}{ }^{-}$ holomorphic $E$-curve $C=C_{1} \cup \cdots \cup C_{n}$ with $g\left(C_{j}\right)=0$ for all $j$.

Let $\tilde{\pi}: \widetilde{M}_{k} \rightarrow \Sigma_{g}$ be the lift of $\pi: M \rightarrow \Sigma_{g}$. Since $\pi$ is $J_{\text {can-holomorphic, }}$ $\tilde{\pi}$ is $\widetilde{J}_{\text {can }}$-holomorphic. Let $h_{j}: S^{2} \rightarrow C_{j}$ be a $\widetilde{J}_{\text {can }}$-holomorphic parametrization of $C_{j}$ and let $l_{j}: S^{2} \rightarrow \mathbb{C}$ be a lift of $\tilde{\pi} \circ h_{j}$ to the universal cover of $\Sigma_{g}$. By Liouville's theorem, $l_{j}$ is constant, and so $\tilde{\pi}\left(C_{j}\right)$ is a point. Since this holds true for all $j$ and since $C$ is connected, $\tilde{\pi}(C)$ is a point too. Hence, 
and since $A$ is the class of a section,

$$
0=\tilde{\pi}_{*}([C])=\tilde{\pi}_{*}(E)=\pi_{*}\left(n_{1} A+n_{2} F\right)=n_{1}\left[\Sigma_{g}\right],
$$

and so $n_{1}=0$, i.e., $E=n_{2} F-\sum_{i=1}^{k} m_{i} E_{i}$. Since the first Chern class of $\widetilde{M}_{k}$ is $\tilde{c}_{1}=2 A+(3-2 g) F-\sum_{i=1}^{k} E_{i}$, the conditions $E \cdot E=-1$ and $\tilde{c}_{1}(E)=1$ become $\sum_{i=1}^{k} m_{i}^{2}=1$ and $2 n_{2}-\sum_{i=1}^{k} m_{i}=1$, which implies $E \in\left\{E_{1}, \ldots, E_{k}, F-E_{1}, \ldots, F-E_{k}\right\}$.

Conversely, $E_{i}$ is clearly an $\widetilde{\omega}_{a b}$-symplectic exceptional class, and the proper transform of the $J_{\text {can }}$-fibre passing through the point $\Theta_{*}\left(E_{i}\right)$ is a $\widetilde{J}_{\text {can }}{ }^{-}$ exceptional rational curve and hence an $\widetilde{\omega}_{a b}$-symplectic exceptional sphere in class $F-E_{i}$.

Finally, we have that $\omega_{a b}(F)=b, c_{1}(F)=2$ and $2 \operatorname{Vol}\left(M, \omega_{a b}\right)=2 a b$. Proposition 2.5 now follows from Theorem 6.A in [5].

\section{Explicit maximal packings in 4 dimensions}

In this section, we realize most of the packing numbers computed in the previous section by explicit symplectic packings. Sometimes, we shall give two different maximal packings. It is known that for the 4 -ball and $\mathbb{C P}^{2}$ and for ruled symplectic 4-manifolds, any two packings by $k$ closed balls of equal size are symplectically isotopic, see $[\mathbf{3}, \mathbf{1 7}]$.

Recall that $\mathbb{R}^{4}$ is endowed with the symplectic form $\omega_{0}=d x_{1} \wedge d y_{1}+$ $d x_{2} \wedge d y_{2}$. We shall often use the Lagrangian splitting $\mathbb{R}^{2}(x) \times \mathbb{R}^{2}(y)$ of $\mathbb{R}^{4}$. Set $\left.\square^{2}(1)=\right] 0,1[\times] 0,1\left[\subset \mathbb{R}^{2}(y)\right.$. In order to construct our symplectic packings by balls, we shall construct explicit symplectic embeddings $\rho_{i}$ of a ball $B^{4}(a)$ into products $U_{i} \times \square^{2}(1)$, where $U_{i} \subset \mathbb{R}^{2}(x)$ is a domain of area just larger than the volume $a^{2} / 2$ of $B^{4}(a)$. The domains $U_{i}$ will look as in Figure 2 below. Each of the symplectic 4 -manifolds $(M, \omega)$ we shall consider contains a domain $W$ such that $\operatorname{Vol}(W, \omega)=\operatorname{Vol}(M, \omega)$ and such that $(W, \omega)$ is explicitly symplectomorphic to $V \times \square^{2}(1)$, where $V$ is a domain in $\mathbb{R}^{2}(x)$. In order to construct an explicit symplectic packing of $(M, \omega)$ by $k$ equal balls it will thus suffice to insert $k$ disjoint domains $U_{i}$ of equal area as in Figure 2 into $V$. Summarizing, our explicit symplectic packings will be of the form

$$
\coprod_{i=1}^{k} B^{4}(a) \stackrel{\amalg \rho_{i}}{\longrightarrow} \coprod_{i=1}^{k} U_{i} \times \square^{2}(1) \stackrel{\amalg \tau_{i} \times \mathrm{id}}{\longrightarrow} V \times \square^{2}(1) \cong W \subset(M, \omega),
$$

where $\tau_{i}$ are translations.

In the explicit packings constructed in $[\mathbf{1 1}, \mathbf{1 2}, \mathbf{1 4}, \mathbf{2 3}]$, a ball is viewed as a product $U \times \square^{2}(1)$, where $U$ is an affine image of a simplex and thus in particular is convex. Our domains $U_{i}$ need not be convex, and so we have a larger arsenal of shapes at our disposal. 
3.1. How to map $\boldsymbol{B}^{4}(\boldsymbol{a})$ to $\boldsymbol{U} \times \square^{2}(\mathbf{1})$. Let $D(a)$ be the open disc in $\mathbb{R}^{2}$ of area $a$ centred at the origin, and let

$$
R(a)=\left\{(x, y) \in \mathbb{R}^{2} \mid 0<x<a, 0<y<1\right\} .
$$

Our symplectic embeddings $B^{4}(a) \hookrightarrow U \times \square^{2}(1) \subset \mathbb{R}^{2}(x) \times \mathbb{R}^{2}(y)$ will be obtained from restricting split symplectomorphisms $\alpha_{1} \times \alpha_{2}: D(a) \times D(a) \rightarrow$ $R(a) \times R(a)$ to $B^{4}(a)$. Notice that in dimension 2 , an embedding is symplectic if and only if it is area and orientation preserving. In order to explicitly describe such embeddings, we follow [20] and start with

Definition 3.1. A family $\mathcal{L}$ of loops in $R(a)$ is admissible if there exists a diffeomorphism $\beta: D(a) \backslash\{0\} \rightarrow R(a) \backslash\{p\}$ for some point $p \in R(a)$ such that

(i) concentric circles are mapped to elements of $\mathcal{L}$,

(ii) in a neighbourhood of the origin $\beta$ is a translation.

Lemma 3.2. Given an admissible family $\mathcal{L}$ of loops in $R(a)$, there exists a symplectomorphism from $D(a)$ to $R(a)$ mapping concentric circles to loops of $\mathcal{L}$.

We refer to $[\mathbf{2 0}]$ for the elementary proof. Notice that the symplectomorphism guaranteed by the lemma is uniquely determined by its image of the ray $\{(x, 0) \in D(a) \mid x \geq 0\}$. We can thus describe a symplectomorphism from $D(a)$ to $R(a)$ pointwise by prescribing an admissible family of loops in $R(a)$ and a smooth line from the centre of $\mathcal{L}$ to the boundary of $R(a)$ meeting each loop exactly once. For the symplectomorphisms $D(a) \rightarrow R(a)$ described in Figure 1, we have chosen this line to be a segment at height

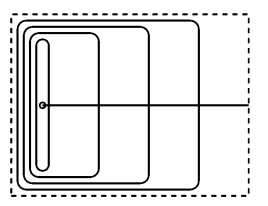

$(\alpha)$

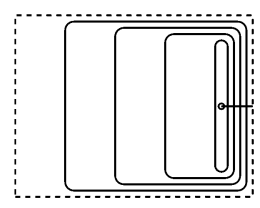

$(\bar{\alpha})$

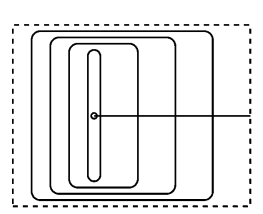

( $\beta)$

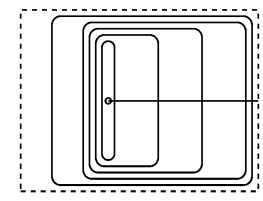

$(\gamma)$

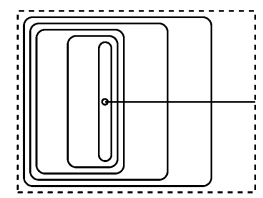

$(\delta)$

Figure 1. Symplectomorphisms $D(a) \rightarrow R(a)$. 
$y=\frac{1}{2}$ from the centre of $\mathcal{L}$ to the right boundary. Consider first the symplectomorphism $\alpha$ represented by $(\alpha)$ in Figure 1 . The image of the restriction of $\alpha \times \alpha$ to $B^{4}(a)$ is contained in the product $U \times \square^{2}(1) \subset \mathbb{R}^{2}(x) \times \mathbb{R}^{2}(y)$, where $U$ is a small neighbourhood of the simplex $[\alpha \alpha]$ of width $a$ shown in Figure 2. In order to see this, we first notice that $B^{4}(a)$ is the union of slices $\coprod\left\{z_{1}\right\} \times D\left(a-\pi\left|z_{1}\right|^{2}\right)$ with $z_{1} \in D(a)$, as well as the union of slices $\coprod D\left(a-\pi\left|z_{2}\right|^{2}\right) \times\left\{z_{2}\right\}$ with $z_{2} \in D(a)$, where $z_{1}=\left(x_{1}, y_{1}\right)$ and $z_{2}=\left(x_{2}, y_{2}\right)$. In view of the definition of $\alpha$, it follows that $(\alpha \times \alpha)\left(B^{4}(a)\right)$ is contained in and close to a set $U \times \square^{2}(1) \subset \mathbb{R}^{2}(x) \times \mathbb{R}^{2}(y)$. To see that $U$ is close to the simplex $[\alpha \alpha]$, notice that for $z \in D(a)$ the $x$-coordinate of $\alpha(z)$ is almost confined to the interval $\left[0, \pi|z|^{2}\right]$. We, therefore, find a small $\epsilon>0$ such that for each $\left(z_{1}, z_{2}\right) \in B^{4}(a)$,

$$
\begin{aligned}
x_{1}\left((\alpha \times \alpha)\left(z_{1}, z_{2}\right)\right)+x_{2}\left((\alpha \times \alpha)\left(z_{1}, z_{2}\right)\right) & =x_{1}\left(\alpha\left(z_{1}\right)\right)+x_{2}\left(\alpha\left(z_{2}\right)\right) \\
& \leq \pi\left|z_{1}\right|^{2}+\epsilon+\pi\left|z_{2}\right|^{2}+\epsilon \\
& <a+2 \epsilon .
\end{aligned}
$$

Since for every neighbourhood $U$ of $[\alpha \alpha]$ we can choose the map $\alpha$ such that $(\alpha \times \alpha)\left(B^{4}(a)\right)$ is contained in $U \times \square^{2}(1)$, we shall work with the simplex $[\alpha \alpha]$ instead of $U$. The bar in the notation $\bar{\alpha}$ used in Figure 1 and Figure 2 indicates that $\bar{\alpha}$ is the mirror of $\alpha$. Figure 2 shows the $x_{1}-x_{2}$-shadows of the image of $B^{4}(a)$ under some other products of the symplectomorphisms in Figure 1 and of their mirrors. We invite the reader to create further shadows.

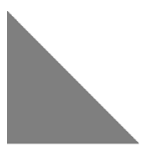

$[\alpha \alpha]$

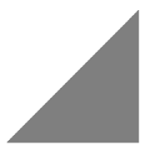

$[\bar{\alpha} \alpha]$

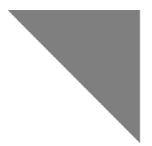

$[\bar{\alpha} \bar{\alpha}]$

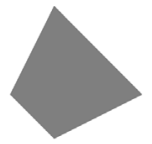

$[\beta \beta]$

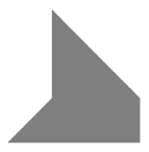

$[\gamma \alpha]$

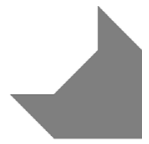

$[\bar{\delta} \delta]$

Figure 2. Some $x_{1}-x_{2}$-shadows.

Remark 3.3. Besides being explicit, the 4-dimensional symplectic packings constructed in $[\mathbf{1 1}, \mathbf{2 3}]$ and in this section have yet another advantage over the packings found in $[\mathbf{5}, \mathbf{6}, \mathbf{1 8}]$. The symplectic packings of $(M, \omega)$ by $k$ balls obtained from the method in $[\mathbf{5}, \mathbf{6}, \mathbf{1 8}]$ are maximal in the following sense. For every $\epsilon>0$ there exists a symplectic embedding $\varphi_{\epsilon}: \coprod_{i=1}^{k} B^{2 n}(a) \hookrightarrow$ $(M, \omega)$ such that

$$
\frac{\operatorname{Vol}\left(\operatorname{Im} \varphi_{\epsilon}, \omega\right)}{\operatorname{Vol}(M, \omega)} \geq p_{k}(M, \omega)-\epsilon
$$

Karshon's symplectic packings of $\left(\mathbb{C P}^{2}, \omega_{S F}\right)$ by 2 and 3 balls $B^{4}\left(\frac{\pi}{2}\right)$ given by the map (3) and compositions of this map with coordinate permutations 
fill exactly $\frac{1}{2}$ and $\frac{3}{4}$ of $\left(\mathbb{C P}^{2}, \omega_{S F}\right)$. Similarly, the 4-dimensional packings in $[\mathbf{2 3}]$ and in this section are maximal in the following sense.

There exists a symplectic embedding $\varphi: \coprod_{i=1}^{k} B^{4}(a) \hookrightarrow(M, \omega)$ such that

$$
\frac{\operatorname{Vol}(\operatorname{Im} \varphi, \omega)}{\operatorname{Vol}(M, \omega)}=p_{k}(M, \omega) \text {. }
$$

Moreover, $\varphi$ is explicit in the following sense. The image $\coprod_{i=1}^{k} \varphi\left(B^{4}(a)\right)$ of $\varphi$ is known, and given $a^{\prime}<a$ one can construct $\varphi$ such that its restriction to $\coprod_{i=1}^{k} B^{4}\left(a^{\prime}\right)$ is given pointwise.

Indeed, choose a sequence $a^{\prime}<a_{j} \nearrow a$. The packings in [23] and our packings $\varphi\left(a_{j}\right): \coprod_{i=1}^{k} B^{4}\left(a_{j}\right) \hookrightarrow(M, \omega)$ can be chosen such that

$$
\operatorname{Im} \varphi\left(a_{j}\right) \subset \operatorname{Im} \varphi\left(a_{j+1}\right) \quad \text { for all } j \text {. }
$$

The claim now follows from a result of McDuff [15], stating that two symplectic embeddings of a closed ball into a larger ball are isotopic via a symplectic isotopy of the larger ball.

3.2. Maximal packings of the 4-ball and $\mathbb{C P}^{2}$. In view of the symplectomorphism (3) and the identity (4) we only need to construct packings of the 4-ball. Consider the standard simplex

$$
\triangle^{2}(\pi)=\left\{0<x_{1}, x_{2} \mid x_{1}+x_{2}<\pi\right\} \subset \mathbb{R}^{2}(x) .
$$

For each $\epsilon>0$, a symplectic embedding of $\triangle^{2}(\pi) \times \square^{2}(1)$ into $B^{4}(\pi+\epsilon)$ can be obtained via the product of the inverse of a map $(\alpha)$ as described in Figure 1. As noticed in [23], an explicit symplectomorphism from $\triangle^{2}(\pi) \times \square^{2}(1)$ to $B^{4}(\pi)$ is given by the "inverse symplectic polar coordinates"

$\left(x_{1}, x_{2}, y_{1}, y_{2}\right) \mapsto\left(\sqrt{\frac{x_{1}}{\pi}} \cos 2 \pi y_{1}, \sqrt{\frac{x_{2}}{\pi}} \cos 2 \pi y_{2}, \sqrt{\frac{x_{1}}{\pi}} \sin 2 \pi y_{1}, \sqrt{\frac{x_{2}}{\pi}} \sin 2 \pi y_{2}\right)$.

In view of the discussion at the beginning of this section, we are left with packing the simplex $\triangle^{2}(\pi)$ with translates of domains of equal area as in Figure 2. It follows from Table 1 that any $k$ of the embeddings in Figure 3(a) yield a maximal packing of $B^{4}$ by $k$ balls, $k=2,3,4$, and that any $k$ of the embeddings in Figure 3(b) yield a maximal packing by $k=5,6$ balls. Figure 3(c) shows a full packing by 9 balls.

Explicit maximal packings of $B^{4}$ by $k \leq 6$ balls were first constructed by Traynor in [23]. Her packings by 5 or 6 balls are constructed by a Lagrangian folding method. Neither Traynor's nor our packing method nor their combination can realize the packing numbers $p_{7}\left(B^{4}\right)=\frac{63}{64}$ and $p_{8}\left(B^{4}\right)=\frac{288}{289}$, but they only fill $\frac{7}{9}$ and $\frac{8}{9}$ of the 4 -ball by 7 and 8 equal balls, respectively.

Question 3.4. Is there an explicit embedding of 7 or 8 equal balls into the 4-ball filling more than $\frac{7}{9}$ and $\frac{8}{9}$ of the volume? 


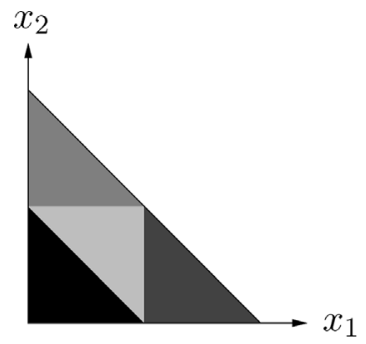

(a)

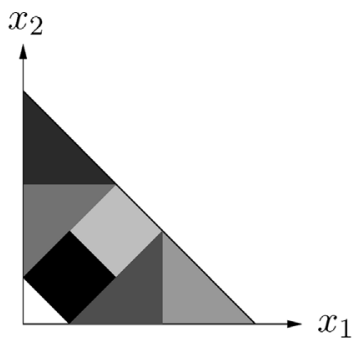

(b)

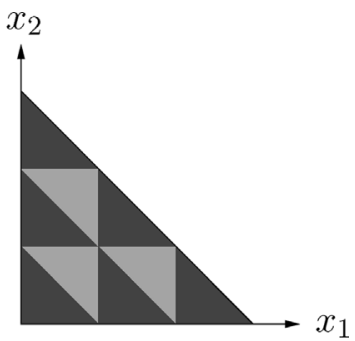

$(c)$

Figure 3. Maximal packings of $B^{4}$ for $k \leq 6$ and $k=l^{2}$.

3.3. Maximal packings of ruled symplectic 4-manifolds. Given a ruled symplectic 4-manifold $\left(M, \omega_{a b}\right)$, let $c_{k}(a, b)$ be the supremum of those $A$ for which $\coprod_{i=1}^{k} B^{2 n}(A)$ symplectically embeds into $\left(M, \omega_{a b}\right)$, so that

$$
p_{k}\left(M, \omega_{a b}\right)=\frac{k c_{k}^{2}(a, b)}{2 \operatorname{Vol}\left(M, \omega_{a b}\right)} .
$$

We shall write $c$ instead of $c_{k}(a, b)$ if $\left(M, \omega_{a b}\right)$ and $k$ are clear from the context.

3.3.1. Maximal packings of $S^{2}(a) \times S^{2}(b)$. As in Proposition 2.1, we assume that $a \geq b$. Represent the symplectic structure of $S^{2}(a) \times S^{2}(b)$ by a split form. Using Lemma 3.2 we symplectically identify $S^{2}(a) \backslash p t$ with ] $0, a[\times] 0,1\left[\right.$ and $S^{2}(b) \backslash p t$ with $] 0, b[\times] 0,1[$. Then

$$
\square(a, b) \times \square^{2}(1)=S^{2}(a) \times S^{2}(b) \backslash\left\{S^{2}(a) \times p t \cup p t \times S^{2}(b)\right\} .
$$

Besides for $k \in\{6,7\}$, we will construct the explicit maximal packings promised after Proposition 2.1 by constructing packings of $\square(a, b) \times \square^{2}(1)$ which realize the packing numbers of $S^{2}(a) \times S^{2}(b)$ computed in Proposition 2.1 and hence are maximal. (It is, in fact, known that all packing numbers of $\square(a, b) \times \square^{2}(1)$ and $S^{2}(a) \times S^{2}(b)$ agree, see [18, Remark 2.1.E]).

To construct explicit maximal packings for all $k$ with $\left\lceil\frac{k}{2}\right\rceil \frac{b}{a} \leq 1$ is a trivial matter. Figure 4 shows a maximal packing by 1 and 2, respectively, 5 and 6 balls.

Assume now that $k=3,4$ and $\frac{b}{a} \geq \frac{1}{2}$. Figure 5 shows maximal packings of $S^{2}(a) \times S^{2}(b)$ by $k$ balls for $\frac{b}{a}=\frac{1}{2}, \frac{b}{a}=\frac{3}{4}$, and $\frac{b}{a}=1$. For $\frac{b}{a}>\frac{1}{2}$, the $\left(x_{1}, x_{2}\right)$-coordinates of the vertices of the "upper left ball" are

$$
(0, c), \quad(a-c, b), \quad(c, c), \quad(a-c, b-c),
$$

where $c=\frac{a+b}{3}$. As in most of the subsequent figures, the three pictures in Figure 5 should be seen as moments of a movie starting at $\frac{b}{a}=\frac{1}{2}$ and ending at $\frac{b}{a}=1$. Each ball in this movie moves in a smooth way. 


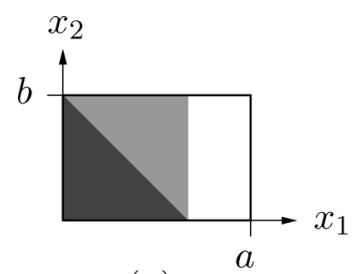

(a)

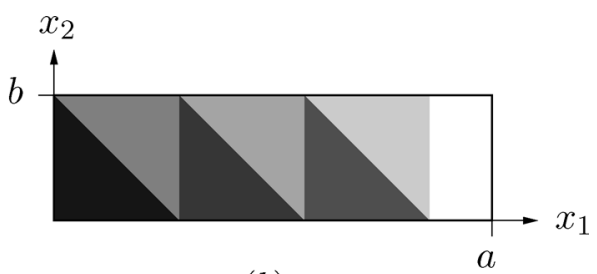

(b)

Figure 4. Maximal packings of $S^{2}(a) \times S^{2}(b)$ by $k$ balls, $\left\lceil\frac{k}{2}\right\rceil \frac{b}{a} \leq 1$.
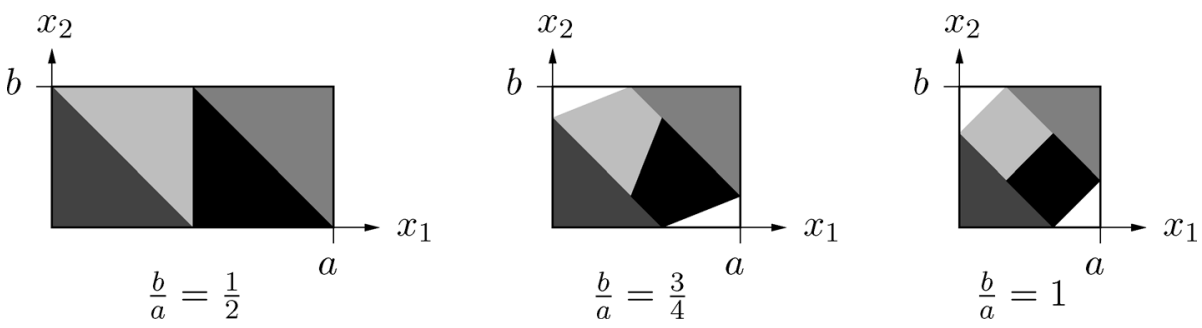

Figure 5. Maximal packings of $S^{2}(a) \times S^{2}(b)$ by 3 and 4 balls, $\frac{b}{a} \geq \frac{1}{2}$.

Next, let $k=5$ and $\frac{b}{a} \geq \frac{1}{3}$. In order to construct a smooth family of maximal packings of $S^{2}(a) \times S^{2}(b)$ by 5 balls, we think of the maximal packing for $\frac{b}{a}=\frac{1}{3}$ rather as in Figure 6 than as in Figure $4(a)$. The $x_{1}$-width of all balls is $\frac{a+2 b}{5}$, and the "upper left ball" has 5 vertices for $\frac{1}{3}<\frac{b}{a} \leq \frac{3}{4}$ and 7 vertices for $\frac{b}{a}>\frac{3}{4}$.

For $k \in\{6,7\}$, we cannot realize the packing numbers $p_{k}\left(S^{2}(a) \times S^{2}(b)\right)$ by directly packing rectangles as for $k \leq 4$. We shall instead construct certain maximal packings of $\mathbb{C P}^{2}$ which correspond to maximal packings of $S^{2}(a) \times S^{2}(b)$. As noticed in [5], the correspondence between symplectic packings and the symplectic blow-up operation and the diffeomorphism mentioned in the proof of Proposition 2.1 imply

Lemma 3.5. Packing $S^{2}(a) \times S^{2}(b)$ by $k$ equal balls $\coprod_{i=1}^{k} B^{4}(c)$ corresponds to packing $\left(\mathbb{C P}^{2},(a+b-c) \omega_{S F}\right)$ by the disjoint union of the $k+1$ balls $B^{4}(a-c) \amalg B^{4}(b-c) \coprod_{i=1}^{k-1} B^{4}(c)$.

In order to make this correspondence plausible, we choose $\frac{b}{a}=\frac{2}{3}$ and $c=c_{6}(a, b)=\frac{a+2 b}{5}$, and we think of $\left(\mathbb{C P}^{2},(a+b-c) \omega_{S F}\right)$ as the simplex of width $a+b-c$ and of $S^{2}(a) \times S^{2}(b)$ as the rectangle of width $a$ and length $b$. As Figure 7 illustrates, the space obtained by removing a ball 

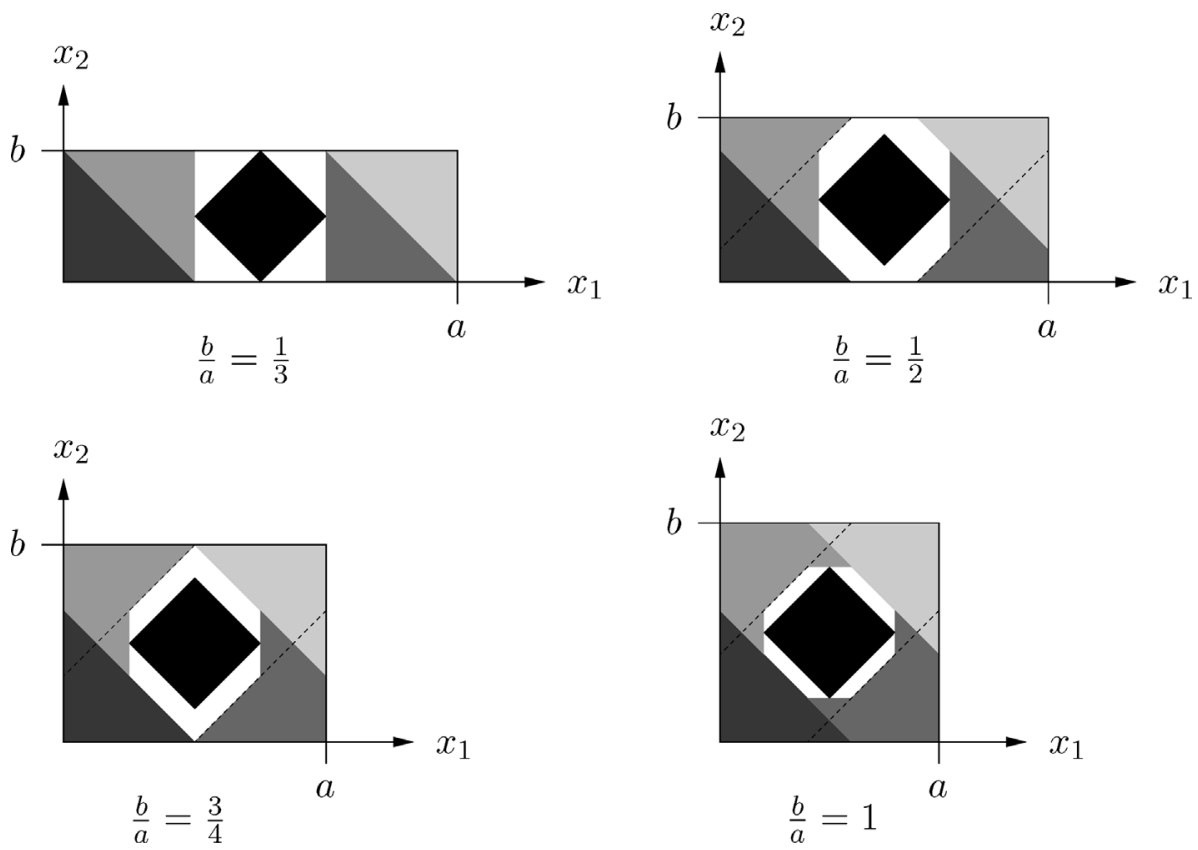

Figure 6. Maximal packings of $S^{2}(a) \times S^{2}(b)$ by 5 balls, $\frac{b}{a} \geq \frac{1}{3}$.

$B^{4}(c)$ from $S^{2}(a) \times S^{2}(b)$ coincides with the space obtained by removing the balls $B^{4}(a-c) \coprod B^{4}(b-c)$ from $\left(\mathbb{C P}^{2},(a+b-c) \omega_{S F}\right)$.

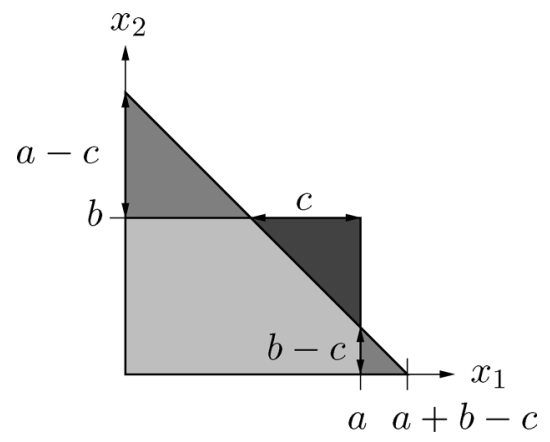

Figure 7. $\left(\mathbb{C P}^{2},(a+b-c) \omega_{S F}\right) \backslash B^{4}(a-c) \bigsqcup B^{4}(b-c)=$ $S^{2}(a) \times S^{2}(b) \backslash B^{4}(c)$.

Figures 8,9 , and 10 describe explicit packings of $\left(\mathbb{C P}^{2},(a+b-c) \omega_{S F}\right)$ by balls $B^{4}(a-c) \coprod B^{4}(b-c) \coprod_{i=1}^{k-1} B^{4}(c)$ for $k \in\{6,7\}$ and $c$ as in Proposition 2.1. The lower left triangle represents $B^{4}(a-c)$ and the black "ball" represents $B^{4}(b-c)$. 

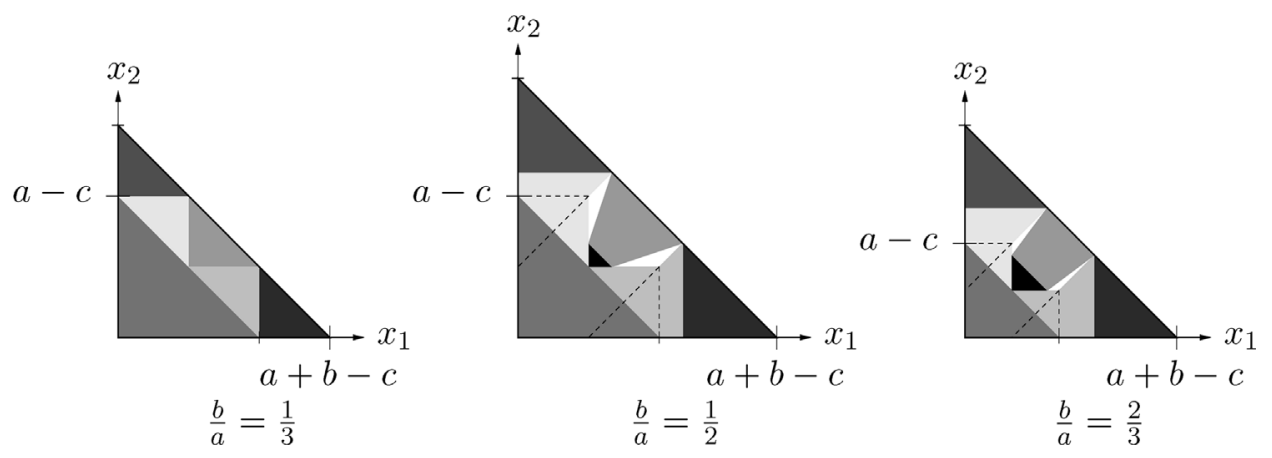

Figure 8. Maximal packings of $S^{2}(a) \times S^{2}(b)$ by 6 balls, $\frac{1}{3} \leq \frac{b}{a} \leq \frac{3}{4}$.
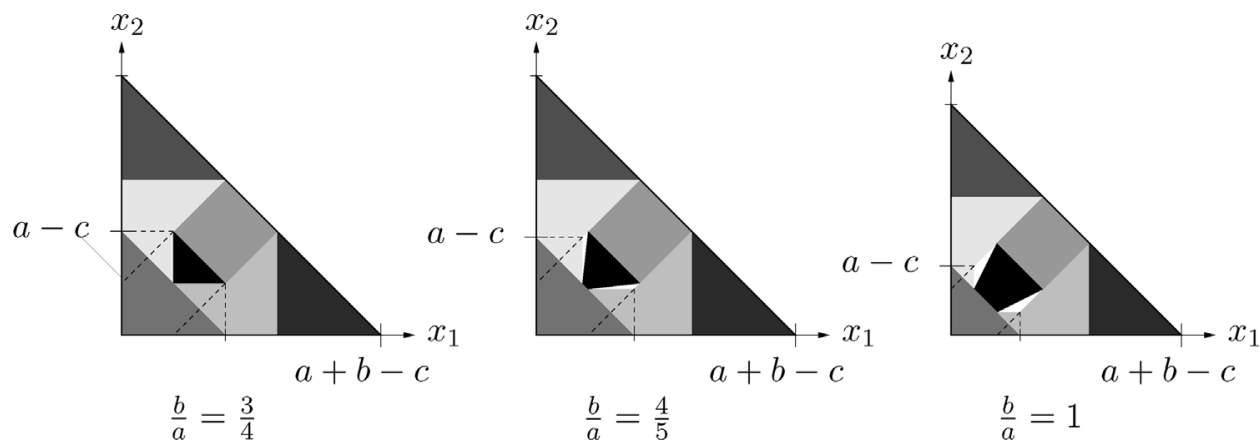

Figure 9. Maximal packings of $S^{2}(a) \times S^{2}(b)$ by 6 balls, $\frac{3}{4} \leq \frac{b}{a} \leq 1$.

From these packings, one obtains explicit packings of $S^{2}(a) \times S^{2}(b)$ as follows. First symplectically blow-up $\left(\mathbb{C P}^{2},(a+b-c) \omega_{S F}\right)$ twice by removing the balls $B^{4}(a-c)$ and $B^{4}(b-c)$ and collapsing the remaining boundary spheres to exceptional spheres in homology classes $D_{1}$ and $D_{2}$. The resulting
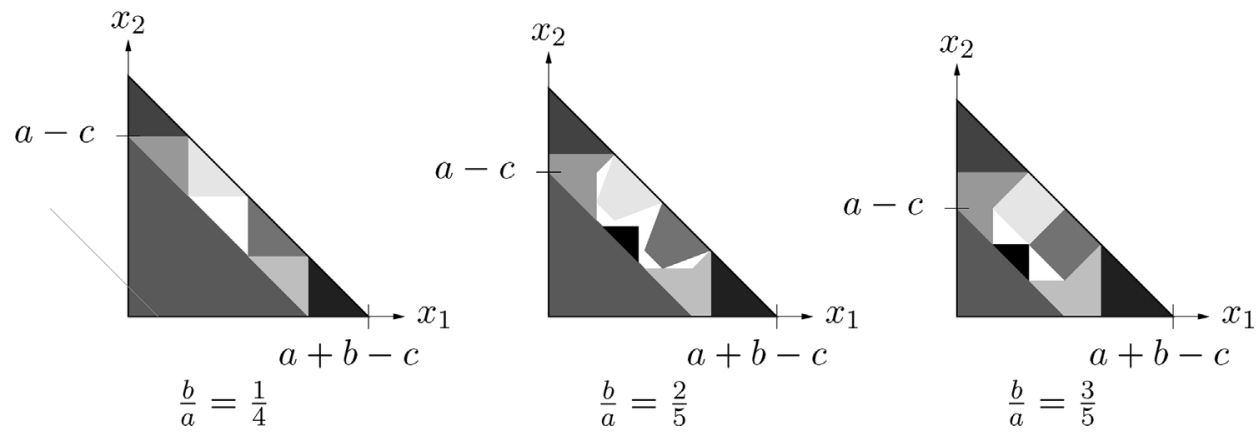

Figure 10. Maximal packings of $S^{2}(a) \times S^{2}(b)$ by 7 balls, $\frac{1}{4} \leq \frac{b}{a} \leq \frac{3}{5}$. 


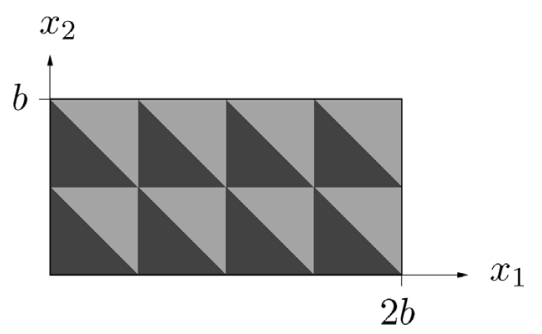

Figure 11. A full packing of $S^{2}(2 b) \times S^{2}(b)$ by 16 balls.

manifold, which is symplectomorphic to $S^{2}(a) \times S^{2}(b)$ blown up at one point with weight $c$, still contains the $k-1$ explicitly embedded balls $B^{4}(c)$, and according to $\left[\mathbf{5}\right.$, Theorem 4.1.A] the exceptional sphere in class $L-D_{1}-D_{2}$ can be symplectically blown down with weight $c$ to yield the $k$ th ball $B^{4}(c)$ in $S^{2}(a) \times S^{2}(b)$.

Finally, the construction of full packings of $S^{2}(m b) \times S^{2}(b)$ by $2 m l^{2}$ balls $(l, m \in \mathbb{N})$ is also straightforward. Figure 11 shows such a packing for $l=m=2$.

3.3.2. Maximal packings of $\left(S^{2} \ltimes S^{2}, \omega_{a b}\right)$. In order to describe our maximal packings of $\left(S^{2} \ltimes S^{2}, \omega_{a b}\right)$, it will be convenient to work with the parameters $\alpha=a-\frac{b}{2}, \beta=b$, so that $\alpha>0, \beta>0$, and $\omega_{a b}=\beta A+(\alpha+\beta) F$. We recall that $S^{2} \ltimes S^{2}$ is diffeomorphic to the blow-up $\widetilde{N}_{1}$ of $\mathbb{C P}^{2}$ at one point via a diffeomorphism under which $L, D_{1}$ correspond to $A+F, A$. We can therefore view $\left(S^{2} \ltimes S^{2}, \omega_{a b}\right)$ as $\widetilde{N}_{1}$ endowed with the symplectic form in class $(\alpha+\beta) L-\alpha D_{1}$ obtained by symplectically blowing up $\left(\mathbb{C P}^{2},(\alpha+\beta) \omega_{S F}\right)$ with weight $\alpha$. Since symplectically blowing up with weight $\alpha$ corresponds to removing a ball $B^{4}(\alpha)$ and collapsing the remaining boundary sphere to an exceptional sphere in class $D_{1}$, we can think of this symplectic manifold as the truncated simplex obtained by removing the simplex of width $\alpha$ from the simplex of width $\alpha+\beta$.

Denote by $\lfloor x\rfloor$ the integer part of $x \geq 0$. In the parameters $\alpha$ and $\beta$, the packings promised after Proposition 2.3 are explicit maximal packings of $\left(S^{2} \ltimes S^{2}, \omega_{a b}\right)$ for all $k$ with $\left\lfloor\frac{k}{2}\right\rfloor \frac{\beta}{\alpha} \leq 1$, for $k \leq 5$ and $\alpha, \beta>0$ arbitrary, and for $k=6$ and $\left.\left.\frac{\beta}{\alpha} \in\right] 0,1\right] \cup\left[4, \infty\left[\right.\right.$. Moreover, given $\omega_{a b}$ with $\frac{\beta}{\alpha}=\frac{l}{m-l}$ for some $l, m \in \mathbb{N}$ with $m>l$, we will construct explicit full packings of $\left(S^{2} \ltimes S^{2}, \omega_{a b}\right)$ by $l(2 m-l)$ balls.

We abbreviate $c_{k}=c_{k}(a, b)=c_{k}\left(S^{2} \ltimes S^{2}, \omega_{a b}\right)$. Using the identity $2 \mathrm{Vol}\left(S^{2} \ltimes S^{2}, \omega_{a b}\right)=\beta(2 \alpha+\beta)$ and (14) we read off from the list in the 
proof of Proposition 2.3 that

$$
\begin{gathered}
\left.c_{1}=\beta, \quad c_{2}=c_{3}=\left\{\beta, \frac{\alpha+\beta}{2}\right\} \text { on }\right] 0,1, \infty[ \\
\left.c_{4}=\left\{\beta, \frac{\alpha+2 \beta}{4}\right\} \text { on }\right] 0, \frac{1}{2}, \infty[ \\
\left.c_{5}=\left\{\beta, \frac{\alpha+2 \beta}{4}, \frac{2 \alpha+2 \beta}{5}\right\} \text { on }\right] 0, \frac{1}{2}, \frac{3}{2}, \infty[ \\
\left.c_{6}=\left\{\beta, \frac{\alpha+3 \beta}{6}, \frac{2 \alpha+3 \beta}{7}, \frac{2 \alpha+2 \beta}{5}\right\} \text { on }\right] 0, \frac{1}{3}, \frac{5}{3}, 4, \infty[.
\end{gathered}
$$

To construct packings with $p_{k}=k \frac{\beta}{2 \alpha+\beta}$ for all $k$ with $\left\lfloor\frac{k}{2}\right\rfloor \frac{\beta}{\alpha} \leq 1$ is very easy. Figure 12(a) shows a maximal packing by 1 ball, and Figures 12(b1) and (b2) show maximal packings by 4 and 5 balls for $\frac{\beta}{\alpha}=\frac{1}{2}$ and $\frac{\beta}{\alpha}<\frac{1}{2}$, respectively. Figure 13 shows maximal packings for $k=2,3$ and $\frac{\beta}{\alpha} \geq 1$.

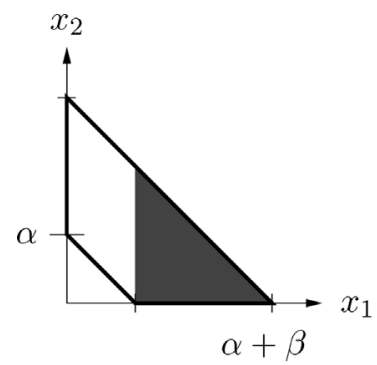

(a)

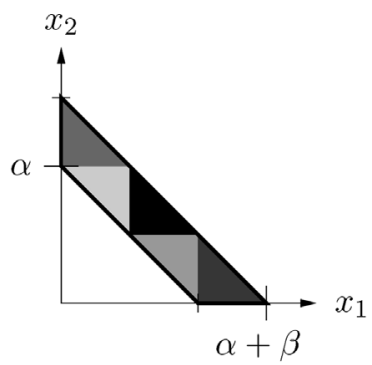

$(b 1)$

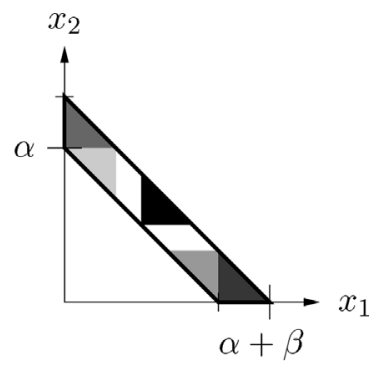

(b2)

Figure 12. Maximal packings of $\left(S^{2} \ltimes S^{2}, \omega_{a b}\right)$ by $k$ balls, $\left\lfloor\frac{k}{2}\right\rfloor \frac{\beta}{\alpha} \leq 1$.

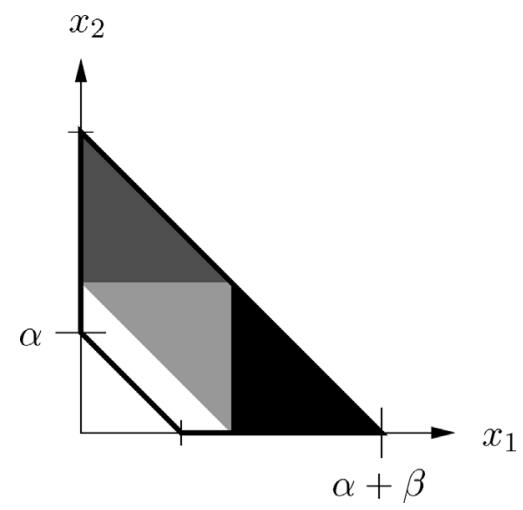

Figure 13. Maximal packings of $\left(S^{2} \ltimes S^{2}, \omega_{a b}\right)$ by 2 and 3 balls, $\frac{\beta}{\alpha} \geq 1$. 

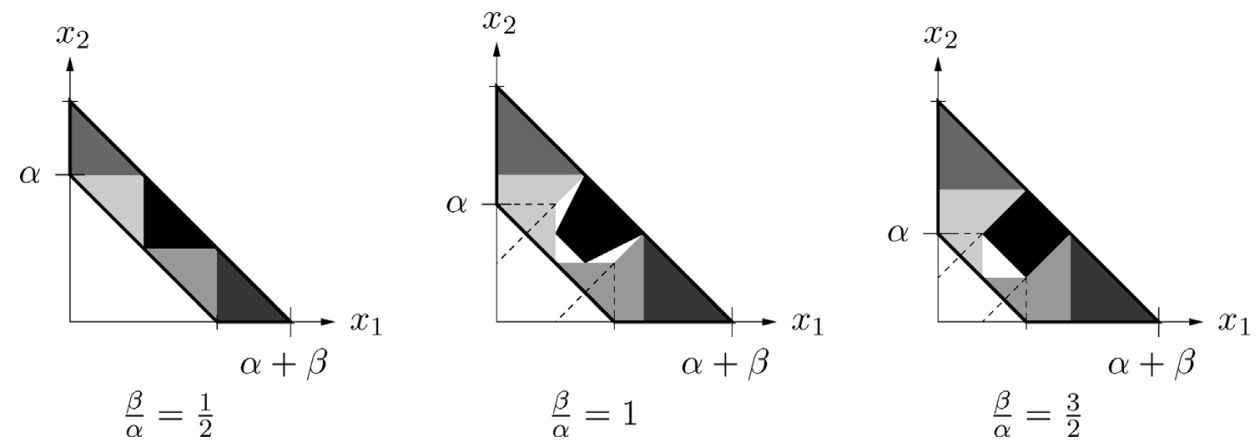

Figure 14. Maximal packings of $\left(S^{2} \ltimes S^{2}, \omega_{a b}\right)$ by 4 and 5 balls, $\frac{1}{2} \leq \frac{\beta}{\alpha} \leq \frac{3}{2}$.

Also our maximal packings by 4 balls are easy to understand (Figure 14 and Figure 15(a)): $2 c_{4}=\beta+\frac{\alpha}{2}$ just means that the two middle grey balls touch each other. As long as $\frac{\beta}{\alpha} \leq \frac{3}{2}$, there is enough room for a fifth (black) ball between these two balls. If $\frac{\beta}{\alpha}>\frac{3}{2}$, there is enough space for a fifth ball if and only if the capacity $c$ of the balls satisfies $2 c+\frac{c}{2} \leq \alpha+\beta$; hence $c_{5}=\frac{2 \alpha+2 \beta}{5}$ (Figures 15(b1) and (b2)).

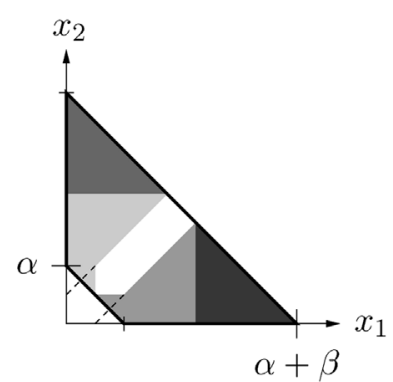

(a) $: \frac{\beta}{\alpha}=3$

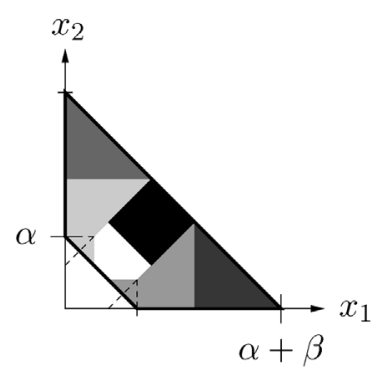

(b1) $: \frac{\beta}{\alpha}=2$

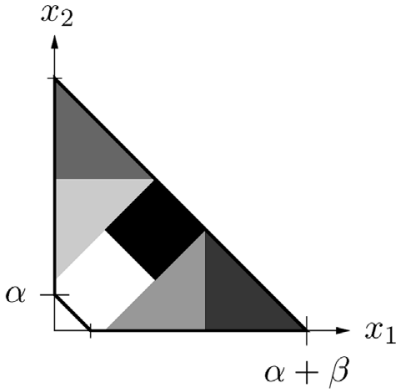

$(b 2): \frac{\beta}{\alpha}=6$

Figure 15. Maximal packings of $\left(S^{2} \ltimes S^{2}, \omega_{a b}\right)$ by 4 and 5 balls, $\frac{\beta}{\alpha} \geq \frac{3}{2}$.

Let now $k=6$. Figure 16 shows maximal packings for $\frac{1}{3} \leq \frac{\beta}{\alpha} \leq 1$. For $\frac{\beta}{\alpha}>\frac{1}{3}$, the vertices of the "lower middle ball" are

$\left(\alpha+\beta-2 c_{6}, c_{6}\right), \quad\left(\frac{\alpha+\beta}{2}, \frac{\alpha+\beta}{2}\right), \quad\left(\alpha+\beta-c_{6}, c_{6}\right), \quad\left(\frac{\alpha+\beta}{2}, \frac{\alpha+\beta}{2}-c_{6}\right)$.

Maximal packings for $\frac{\beta}{\alpha} \geq 4$ are illustrated in Figure 17 .

Remark 3.6. It is not a coincidence that we were not able to construct maximal packings of $\left(S^{2} \ltimes S^{2}, \omega_{a b}\right)$ by 6 balls for all ratios $\frac{\beta}{\alpha}>0$. Indeed, 

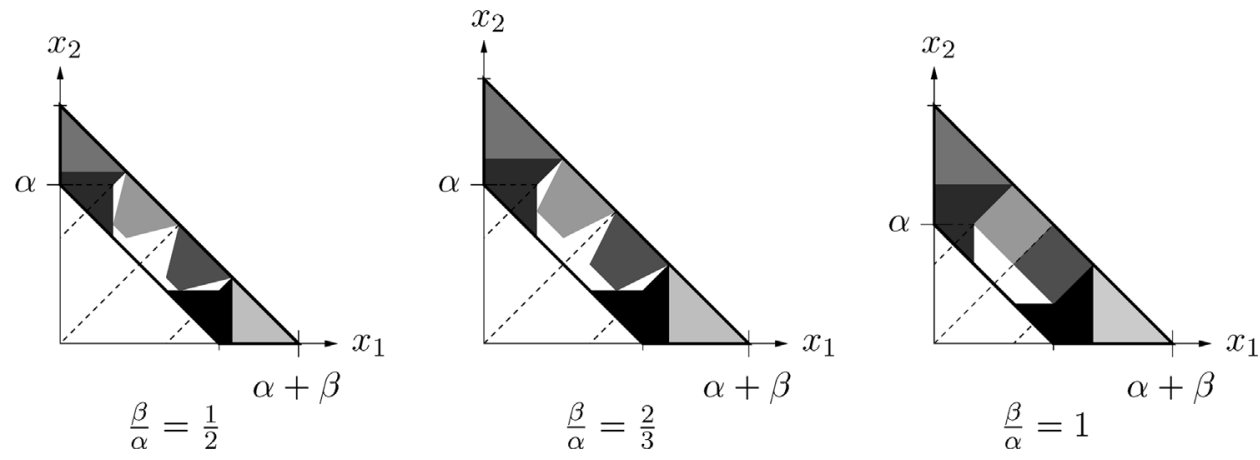

Figure 16. Maximal packings of $\left(S^{2} \ltimes S^{2}, \omega_{a b}\right)$ by 6 balls, $\frac{1}{3} \leq \frac{\beta}{\alpha} \leq 1$.
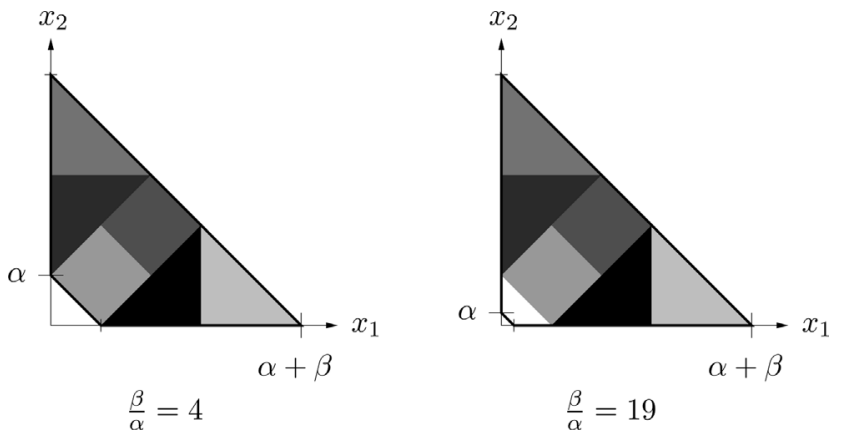

Figure 17. Maximal packings of $\left(S^{2} \ltimes S^{2}, \omega_{a b}\right)$ by 6 balls, $\frac{\beta}{\alpha} \geq 4$.

a maximal packing of $\left(S^{2} \ltimes S^{2}, \omega_{a b}\right)$ by 6 equal balls for $\frac{\beta}{\alpha}=\frac{5}{3}$ corresponds to a maximal packing of the 4 -ball by 7 equal balls.

Finally, suppose that $\frac{\beta}{\alpha}=\frac{l}{m-l}$ for some $l, m \in \mathbb{N}$ with $m>l$. We can then fill $\left(S^{2} \ltimes S^{2}, \omega_{a b}\right)$ by $l(2 m-l)$ balls by decomposing $S^{2} \ltimes S^{2}$ into $l$ shells and filling the $i$ th shell with $2 m+1-2 i$ balls (see Figure 18, where $l=2$ and $m=4$ ).

3.3.3. Maximal packings of $\Sigma_{g}(a) \times S^{2}(b)$ and $\left(\Sigma_{g} \ltimes S^{2}, \omega_{a b}\right)$ for $\boldsymbol{g} \geq \mathbf{1}$. Fix $a>0$ and $b>0$. We represent the symplectic structure of $\Sigma_{g}(a) \times S^{2}(b)$ by a split form. Removing a wedge of $2 g$ loops from $\Sigma(a)$ and a point from $S^{2}(b)$, we see that $\Sigma_{g}(a) \times S^{2}(b)$ contains $\square(a, b) \times \square^{2}(1)$. The explicit construction of the "standard Kähler form" in class $\left[\omega_{a b}\right]$ given in [16, Section 3] and [19, Exercise 6.14] shows that also $\left(\Sigma_{g} \ltimes S^{2}, \omega_{a b}\right)$ endowed with this standard form contains $\square(a, b) \times \square^{2}(1)$. The explicit maximal packings promised after Proposition 2.5 can thus be constructed as for $S^{2}(a) \times S^{2}(b)$, see Figures 4 and 11 . 


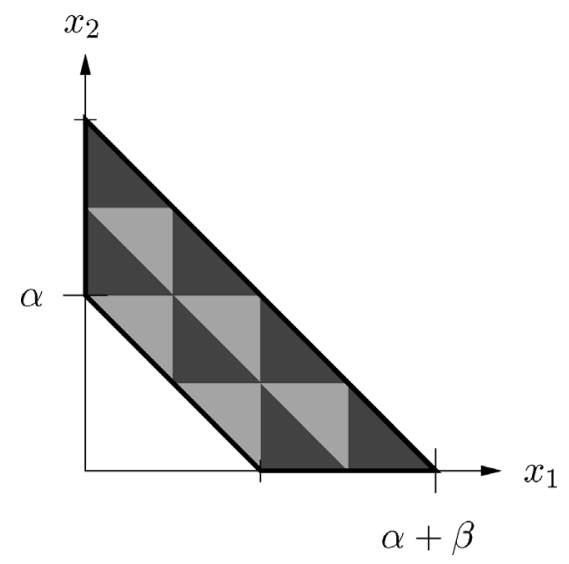

Figure 18. A full packing of $\left(S^{2} \ltimes S^{2}, \omega_{a b}\right), \frac{\beta}{\alpha}=1$, by 12 balls.

3.4. Maximal packings of 4-dimensional ellipsoids. Some explicit maximal packings of 4-dimensional ellipsoids were found in $[\mathbf{1 4}, \mathbf{2 3}]$. Our embedding method can be used to recover their packing results. We refer the interested reader to Section 4.5 of $[\mathbf{2 1}]$.

\section{Explicit maximal packings of the $2 n$-ball and of $\mathbb{C P}^{n} \backslash \mathbb{R} \mathbb{P}^{n}$}

In dimensions $2 n \geq 6$, only few maximal symplectic packings by equal balls are presently known. We refer to Section 5 of $[\mathbf{2 1}]$ for an overview. In this section, we provide explicit maximal packings of $B^{2 n}$ and $\mathbb{C P}^{n}$ by $k \leq 2^{n}$ and $k=l^{n}$ balls and of $B^{2 n} \backslash \mathbb{R}^{n}(x)$ and $\mathbb{C P}^{n} \backslash \mathbb{R} \mathbb{P}^{n}$ by $k \leq 2^{n}$ and $k=2^{l n}$ balls.

4.1. Maximal packings of the $2 \boldsymbol{n}$-ball and $\mathbb{C P}^{n}$. Recall that for $n \geq 3$ the known packing numbers of $B^{2 n}$ and $\left(\mathbb{C P}^{n}, \omega_{S F}\right)$ are given by (6) and (7). An explicit maximal packing of $\mathbb{C P}^{n}$ by $k \leq n+1$ balls was found by Karshon in [11], and explicit full packings of $B^{2 n}$ by $l^{n}$ balls for each $l \in \mathbb{N}$ were given by Traynor in $[\mathbf{2 3}]$. Taking $l=2$, any $k$ balls of such a packing yield a maximal packing by $k$ balls. The following different construction of an explicit full packing of $B^{2 n}$ by $l^{n}$ equal balls is mentioned in [23, Remark 5.13]. Set

$$
\begin{aligned}
& \triangle^{n}(a)=\left\{0<x_{1}, \ldots, x_{n} \mid \sum_{i=1}^{n} \frac{x_{i}}{a}<1\right\} \subset \mathbb{R}^{n}(x), \\
& \square^{n}(a)=\left\{0<y_{i}<a, 1 \leq i \leq n\right\} \subset \mathbb{R}^{n}(y) .
\end{aligned}
$$

We see as in Section 3.1 that we can think of $B^{2 n}(a)$ as $\triangle^{n}(a) \times \square^{n}(1)$. An explicit symplectomorphism $\triangle^{n}(a) \times \square^{n}(1) \rightarrow B^{2 n}(a) \subset \mathbb{R}^{n}(x) \times \mathbb{R}^{n}(y)$ is 
given by the "inverse symplectic polar coordinates"

$$
\begin{array}{r}
\left(x_{1}, \ldots, x_{n}, y_{1}, \ldots, y_{n}\right) \mapsto \quad\left(\sqrt{\frac{x_{1}}{\pi}} \cos 2 \pi y_{1}, \ldots, \sqrt{\frac{x_{n}}{\pi}} \cos 2 \pi y_{n},\right. \\
\left.\sqrt{\frac{x_{1}}{\pi}} \sin 2 \pi y_{1}, \ldots, \sqrt{\frac{x_{n}}{\pi}} \sin 2 \pi y_{n}\right) .
\end{array}
$$

In particular, we can think of $B^{2 n}\left(\frac{\pi}{l}\right)$ as $\triangle^{n}\left(\frac{\pi}{l}\right) \times \square^{n}(1)$ and of $B^{2 n}(\pi)$ as $\triangle^{n}(\pi) \times \square^{n}(1)$. The matrix $\operatorname{diag}\left[l, \ldots, l, \frac{1}{l}, \ldots, \frac{1}{l}\right] \in \operatorname{Sp}(n ; \mathbb{R})$ maps $\triangle^{n}\left(\frac{\pi}{l}\right) \times$ $\square^{n}(1)$ to $\triangle^{n}(\pi) \times \square^{n}\left(\frac{1}{l}\right)$. It is clear how to insert $l^{n}$ copies of $\triangle^{n}(\pi) \times \square^{n}\left(\frac{1}{l}\right)$ into $\triangle^{n}(\pi) \times \square^{n}(1)$. Yet another full packing of $B^{2 n}$ by $l^{n}$ balls is described in Section 5 .

4.2. Maximal packings of $\boldsymbol{B}^{2 n} \backslash \mathbb{R}^{n}(\boldsymbol{x})$ and $\mathbb{C P}^{n} \backslash \mathbb{R} \mathbb{P}^{n}$. Let $\square^{n}(1)=$ $\square^{n}(1) \backslash(1 / 2, \ldots, 1 / 2)$ be the cube $\square^{n}(1)$ without its centre. The inverse symplectic polar coordinates embed $\triangle^{n}(\pi) \times \square^{n}(1)$ into $B^{2 n}(\pi) \backslash \mathbb{R}^{n}(x)$. Recall now that we can think of $B^{2 n}\left(\frac{\pi}{2^{l}}\right)$ as $\triangle^{n}\left(\frac{\pi}{2^{l}}\right) \times \square^{n}(1)$, which is symplectomorphic to $\triangle^{n}(\pi) \times \square^{n}\left(\frac{1}{2^{l}}\right)$. It is clear how to insert $2^{l n}$ copies of this set into $\triangle^{n}(\pi) \times \nabla^{n}(1)$.

Remark 4.1. Karshon's explicit packing of $\left(\mathbb{C P}^{n}, \omega_{S F}\right)$ by $k \leq n+1$ balls is maximal in the sense of (14). Since in dimensions $\geq 6$, it is not yet known whether the space of symplectic embeddings of a closed ball into a larger ball is connected, all other explicit (and nonexplicit) maximal symplectic packings known in dimensions $\geq 6$ are maximal only in the sense of (15).

\section{Relative maximal packings of $\left(\mathbf{B}^{2 n}, \mathbf{B}^{2 n} \cap \mathbb{R}^{n}(x)\right)$ and $\left(\mathbb{C P}^{n}, \mathbb{R P}^{n}\right)$}

In Section 3, we have constructed explicit maximal packings of $B^{4}$ and $\mathbb{C P}^{2}$ by $k \leq 6$ and $k=l^{2}$ balls. In this section, we verify that these packings are relative packings for the Lagrangian submanifolds $B^{4} \cap \mathbb{R}^{2}(x)$ and $\mathbb{R P}^{2}$. We shall also construct explicit maximal packings of $B^{2 n}$ and $\mathbb{C P}^{n}, 2 n \geq 6$, by $k \leq 2^{n}$ and $k=l^{n}$ balls which are relative packings for $B^{2 n} \cap \mathbb{R}^{n}(x)$ and $\mathbb{R} \mathbb{P}^{n}$. For these $n$ and $k$, we thus find

$$
p_{k}\left(B^{2 n}, B^{2 n} \cap \mathbb{R}^{n}(x)\right)=p_{k}\left(B^{2 n}\right) \quad \text { and } \quad p_{k}\left(\mathbb{C P}^{n}, \mathbb{R P}^{n}\right)=p_{k}\left(\mathbb{C P}^{n}\right)
$$

Since the symplectomorphism (4) maps $B^{2 n} \cap \mathbb{R}^{n}(x)$ to $\mathbb{R P}^{n}$ and since $p_{k}\left(B^{2 n}\right)=p_{k}\left(\mathbb{C P}^{n}\right)$, we only need to consider $\left(B^{2 n}, B^{2 n} \cap \mathbb{R}^{n}(x)\right)$.

To begin with, we note that $p_{k}\left(B^{2}, B^{2} \cap \mathbb{R}(x)\right)=1$ for all $k \geq 1$. This follows from an appropriate version of Lemma 3.2. 
Let now $n=2$. We recall that our explicit maximal packings of $B^{4}$ were of the form

$$
\coprod_{i=1}^{k} B^{4}(a) \stackrel{\amalg \rho_{i}}{\longrightarrow} \coprod_{i=1}^{k} U_{i} \times \square^{2}(1) \stackrel{\amalg \tau_{i} \times \mathrm{id}}{\longrightarrow} \triangle^{2}(\pi) \times \square^{2}(1) \stackrel{\sigma}{\longrightarrow} B^{4}(\pi),
$$

where each $U_{i}$ is a (neighbourhood of an) $x_{1}-x_{2}$-shadow as in Figure 2, the $\tau_{i}$ are translations, and $\sigma$ is given by inverse symplectic polar coordinates as in Section 3.2. If we choose the symplectomorphisms $D(a) \rightarrow R(a)$ constructed in Section 3.1 and described by drawings as in Figure 1 in such a way that concentric circles are mapped to curves symmetric to the axis $\{y=1 / 2\}$, then the set $D(a) \cap \mathbb{R}(x)=\{(x, y) \in D(a) \mid y=0\}$ is mapped into $(0, a) \times\{1 / 2\}$. A map $\rho_{i}$ obtained by restricting the product of two such symplectomorphisms to $B^{4}(a)$, therefore, maps $B^{4}(a) \cap \mathbb{R}^{2}(x)$ into $U_{i} \times\{*\}$, where $*=(1 / 2,1 / 2) \in \mathbb{R}^{2}(y)$. Since $\sigma$ maps $\triangle^{2}(\pi) \times\{*\}$ to $\mathbb{R}^{n}(x)$, we indeed obtain relative symplectic packings of $\left(B^{4}, B^{4} \cap \mathbb{R}^{2}(x)\right)$.

The maximal packing of $B^{2 n}$ by $l^{n}$ balls constructed in Section 4.1 does not provide a relative packing of $\left(B^{2 n}, B^{2 n} \cap \mathbb{R}^{n}(x)\right)$. In order to describe a relative packing, we denote by $\diamond^{n}(a)$ the interior of the dual to the cube $\square^{n}(a) \subset \mathbb{R}^{n}(x)$, i.e., the interior of the convex hull of the midpoints of the $2 n$ faces of $\square^{n}(a)$. Two translates of $\diamond^{2}(a)$ appear in Figure $3(b)$, and $\diamond^{3}(a)$ is the regular octahedron of height $a$. Consider now the symplectomorphism $\mu: D^{2}(a) \rightarrow R(a)$ described by Figure 19. To be precise, we require that the centre of $D(a)$ is mapped to the centre $(a / 2,1 / 2)$ of $R(a)$, that the segment $\{(x, 0) \mid 0 \leq x<\sqrt{a / \pi}\}$ is mapped to the segment $\{(x, 1 / 2) \mid a / 2 \leq x<a\}$, and that concentric circles are mapped to curves which are symmetric to both axes $\{x=a / 2\}$ and $\{y=1 / 2\}$. With this choice of $\mu$, the $n$-fold product $\mu \times \cdots \times \mu$ maps $B^{2 n}(a)$ to a set contained in and close to $U \times \square^{n}(1)$, where $U$ is an arbitrarily small neighbourhood of $\diamond^{n}(a)$. Moreover, $\mu \times \cdots \times \mu$ maps $B^{2 n}(a) \cap \mathbb{R}^{n}(x)$ into $U \times\{*\}$, where $*=(1 / 2, \ldots, 1 / 2)$. Conversely, the inverse of $\mu \times \cdots \times \mu$ provides a symplectic embedding of $\nabla^{n}(a) \times \square^{n}(1)$ into an arbitrarily small neighbourhood of $B^{2 n}(a)$, which maps $\diamond^{n}(a) \times\{*\}$ to $\mathbb{R}^{n}(x)$. We can thus think of $B^{2 n}\left(\frac{\pi}{l}\right)$ as $\diamond^{n}\left(\frac{\pi}{l}\right) \times \square^{n}(1)$ and of $B^{2 n}(\pi)$

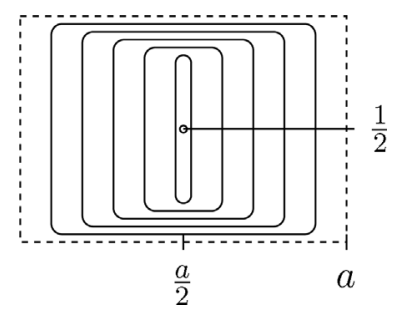

Figure 19. The symplectomorphism $\mu: D(a) \rightarrow R(a)$. 
as $\diamond^{n}(\pi) \times \square^{n}(1)$. It is clear how to insert $l^{n}$ copies of $\diamond^{n}\left(\frac{\pi}{l}\right) \times \square^{n}(1)$ into $\diamond^{n}(\pi) \times \square^{n}(1)$ such that $\diamond^{n}\left(\frac{\pi}{l}\right) \times\{*\}$ is mapped to $\diamond^{n}(\pi) \times\{*\}$.

\section{References}

[1] J.-F. Barraud and O. Cornea, Homotopical dynamics in symplectic topology, math.SG/0506355.

[2] J.-F. Barraud and O. Cornea, Lagrangian intersections and the Serre spectral sequence, math.DG/0401094.

[3] P. Biran, Connectedness of spaces of symplectic embeddings, Int. Math. Res. Notices (1996), 487-491.

[4] P. Biran, The geometry of symplectic packing, Ph.D. thesis, Tel-Aviv University, 1997.

[5] P. Biran, Symplectic packing in dimension 4, Geom. Funct. Anal. 7 (1997), 420437.

[6] P. Biran, A stability property of symplectic packing, Invent. Math. 136 (1999), $123-155$.

[7] P. Biran, Lagrangian barriers and symplectic embeddings, Geom. Funct. Anal. 11 (2001), 407-464.

[8] P. Biran, From symplectic packing to algebraic geometry and back, in European congress of mathematics, Vol. II (Barcelona, 2000), Progr. Math. 202 (2001), 507-524, Birkhäuser, Basel.

[9] M. Demazure, Surfaces de del Pezzo II-V, in Séminaire sur les Singularités des Surfaces (1976-1977), Lecture Notes Math. 777, pp. 23-69, Springer-Verlag, 1980.

[10] M. Gromov, Pseudo-holomorphic curves in symplectic manifolds, Invent. Math. 82 (1985), 307-347.

[11] Y. Karshon, Appendix to [18]. Invent. Math. 115 (1994), 431-434.

[12] B. Kruglikov, A remark on symplectic packings, Dokl. Akad. Nauk 350 (1996), 730-734.

[13] F. Lalonde and D. McDuff, The classification of ruled symplectic 4-manifolds, Math. Res. Lett. 3 (1996), 769-778.

[14] F. M. Maley, J. Mastrangeli and L. Traynor, Symplectic packings in cotangent bundles of tori, Exp. Math. 9 (2000), 435-455.

[15] D. McDuff, Blowing up and symplectic embeddings in dimension 4, Topology 30 (1991), 409-421.

[16] D. McDuff, An irrational ruled symplectic 4-manifold, The Floer memorial volume, 545-554, Progr. Math. 133. Birkhäuser, Basel, 1995.

[17] D. McDuff, From symplectic deformation to isotopy, Topics in symplectic 4manifolds (Irvine, CA, 1996), 85-99, First Int. Press Lect. Ser., I, Internat. Press, Cambridge, MA, 1998.

[18] D. McDuff and L. Polterovich, Symplectic packings and algebraic geometry, Invent. math. 115 (1994), 405-429. 
[19] D. McDuff and D. Salamon, Introduction to Symplectic Topology, Oxford Math. Monographs, 2nd ed., The Clarendon Press, Oxford University Press, New York, 1998.

[20] F. Schlenk, Symplectic embeddings of ellipsoids, Israel J. Math. 138 (2003), 215252 .

[21] F. Schlenk, Packing symplectic manifolds by hand, math.SG/0409568.

[22] C. Taubes, $S W \Rightarrow$ Gr: From the Seiberg-Witten equations to pseudo-holomorphic curves, J. Amer. Math. Soc. 9 (1996), 845-918.

[23] L. Traynor, Symplectic packing constructions, J. Diff. Geom. 42 (1995), 411-429.

DÉPARTEMEnt de MathÉmatiques

Université LiBre de Bruxelles

CP 218, Boulevard du Triomphe,

1050 BRuXelles, Belgium

E-mail address: fschlenk@ulb.ac.be

Received 12/15/2004, accepted 9/9/2005

These pictures were painted in 1998 at ETH Zürich. The words were added in 2004 at Leipzig University. I thank both institutions for their support and Peter Albers, Paul Biran and Octav Cornea for valuable discussions. 